\title{
Spatial variation of the fine-structure parameter and the cosmic microwave background
}

\author{
Kris Sigurdson, ${ }^{*}$ Andriy Kurylov, ${ }^{\dagger}$ and Marc Kamionkowski ${ }^{\dagger}$ \\ California Institute of Technology, Mail Code 130-33, Pasadena, California 91125, USA
}

(Received 19 June 2003; published 19 November 2003)

\begin{abstract}
We study the effects on cosmic microwave background (CMB) temperature and polarization anisotropies of spatial fluctuations of the fine-structure parameter between causally disconnected regions of the Universe at the time of recombination. Analogous to weak gravitational lensing, in addition to modifying the mean power spectra and inducing a curl component ( $B$ mode) to the polarization, spatial fluctuations of the fine-structure parameter induce higher-order (non-Gaussian) temperature and polarization correlations in the CMB. We calculate these effects for the general case of arbitrary correlation between temperature fluctuations and finestructure parameter fluctuations, and show the results for a model where these two types of fluctuations are uncorrelated. The formalism we present here may also be applied to other modifications of recombination physics that do not significantly alter the evolution of the dominant density perturbations. We discuss the constraints on the effective Lagrangian for the variable fine-structure parameter necessary to realize this scenario.
\end{abstract}

DOI: 10.1103/PhysRevD.68.103509

PACS number(s): 98.80.Es, 06.20.Jr, 98.70.Vc

\section{INTRODUCTION}

The possibility that the fine-structure parameter $\alpha$ may vary in time has long been entertained [1-8], and has received renewed interest with recent evidence from quasar spectra that may support a variation of less than one part in $10^{4}$ over a time scale of $\sim 10 \mathrm{Gyr}[9,10]$. Although the results may still be controversial, the observational work has inspired theoretical work that investigates models with variable $\alpha[11-18]$, as well as other work that investigates possible connections with dark energy and new long-range forces [19-21]. It has also stimulated a more careful reinvestigation of the constraints placed on variable $\alpha$ from bigbang nucleosynthesis [22].

Of course, if a relativistic theory allows for temporal variation of $\alpha$ then it must also allow for spatial variations of $\alpha$ between regions not in causal contact. In this paper we study cosmological probes of spatial variations in $\alpha$, focusing in particular on the cosmic microwave background (CMB), which is rapidly becoming an increasingly precise probe of cosmological models [23-25], as well as the physics that underlies them [26]. As we will see, spatial variation in $\alpha$ induces a spatially varying power spectrum. This induces "non-Gaussian" signatures in the CMB, in the form of locally anisotropic correlation functions, that cannot be described by the power spectrum alone (although strictly speaking the joint probability distribution of temperatures at $n$ points remains a multivariate Gaussian). Quite interestingly, these effects are analogous to those of weak gravitational lensing ("cosmic shear") on the CMB, but differ in detail. So, for example, spatial variation of $\alpha$ can alter slightly the CMB power spectrum, induce a curl component ( $B$ mode) in the polarization, and induce higher-order temperature and polarization correlations in the CMB. Effects

\footnotetext{
*Electronic address: ksigurds@tapir.caltech.edu

†Electronic address: kurilov@krl.caltech.edu

‡Electronic address: kamion@ tapir.caltech.edu
}

such as those we investigate here may also arise if there are other spatial variations in recombination physics that do not involve significant density or pressure perturbations. Our calculations are thus illustrative theoretically, apart from the specific application on which we focus.

Below, we first review previous work $[27,28]$ that shows how the recombination history depends on a homogeneous shift in $\alpha$ and then discuss how it affects the CMB power spectrum. We then show how spatial variations of $\alpha$, in which the mean value of $\alpha$ is unaltered, affect the CMB temperature and polarization power spectra, and in so doing show that a curl component is induced in the CMB polarization. We then calculate the CMB bispectrum and trispectrum induced by spatial $\alpha$ variation. Throughout, we compare with the analogous calculations for weak lensing, and show how the effects of weak lensing and spatial $\alpha$ variations differ. We then discuss the properties of and constraints on a toy field-theory model for spatial $\alpha$ variation that produces the CMB effects we investigate here, without inducing significant density perturbations.

Before proceeding further, we clarify that here we investigate spatial variations in the fine-structure parameter $\alpha$ $=e^{2} / \hbar c$ that arise only from spatial variations in the electromagnetic gauge coupling $e$; we do not tinker with relativity nor quantum mechanics.

\section{RECOMBINATION AND $\alpha$}

Recombination depends on the value of $\alpha$ because the visibility function, the probability distribution of when a photon last scattered, is dependent on $\alpha$. The visibility function is defined as

$$
g(t)=e^{-\tau} \frac{d \tau}{d t}
$$

where

$$
\frac{d \tau}{d t}=x_{e} n_{p} c \sigma_{T}
$$


is the differential optical depth of photons due to Thomson scattering. Here,

$$
\sigma_{T}=\frac{8 \pi \hbar^{2} \alpha^{2}}{3 m_{e}^{2} c^{2}}
$$

is the Thomson cross section, $n_{p}$ is the total number density of protons (both free and bound), and $x_{e}$ is the fraction of free electrons. The strongest effect of variations of $\alpha$ on this quantity occur due to the alteration of the ionization history $x_{e}(t)$.

The recombination of hydrogen cannot proceed through direct recombination to the ground state because the emitted photon will immediately ionize a neighboring atom with high probability. Instead, the ionized fraction decreases mainly through the two-photon process $2 s \rightarrow 1 s$, or via the cosmological redshifting of $2 p \rightarrow 1 s$ Lyman- $\alpha$ photons out of the Lyman- $\alpha$ line. These processes are described by a single differential equation [29],

$$
\frac{d x_{e}}{d t}=\mathcal{C}\left[\beta e^{-\left(B_{1}-B_{2}\right) / k_{B} T}\left(1-x_{e}\right)-\mathcal{R} n_{p} x_{e}^{2}\right]
$$

where $\beta$ is the ionization coefficient, $\mathcal{R}$ is the recombination coefficient, $\mathcal{C}$ is the Peebles efficiency factor (discussed below), and

$$
B_{n}=\frac{m_{e} c^{2} \alpha^{2}}{2 n^{2}}
$$

is the binding energy of the level with principle quantum number $n$.

Through the Einstein relations we relate $\beta$ to $\mathcal{R}$ through

$$
\beta=\left(\frac{m_{e} k_{B} T}{2 \pi \hbar^{2}}\right)^{3 / 2} e^{-B_{2} / k_{B} T} \mathcal{R} .
$$

The recombination coefficient can be written

$$
\mathcal{R}=\sum_{n=2}^{\infty} \sum_{l=0}^{n-1} \alpha_{n l} w_{n}
$$

where

$$
\alpha_{n l}=\frac{8 \pi(2 l+1)}{\left(2 \pi m_{e} k_{B} T\right)^{3 / 2} c^{2}} e^{B_{n} / k_{B} T} \int_{B_{n}}^{\infty} d(h \nu) \sigma_{n l}^{b f} \frac{(h \nu)^{2}}{e^{h \nu / k_{B} T}-1},
$$

is the rate at which atoms recombine to the $n, l$ energy level and $w_{n}$ is the efficiency for an $n$ level to survive in a plasma [30]. The details of $w_{n}$ are not important for the present discussion, other than to note that at the densities of interest it is unity for $n<n_{\max } \sim 500$. Since the dominant contributions to $\mathcal{R}$ come from $n \lesssim 50, \mathcal{R}$ is insensitive to the weak $\alpha$ dependence of $n_{\max }$. Above, $\alpha_{n l}$ is written in terms of the ionization cross section $\sigma_{n l}^{b f}$, which can be expressed in the form [31]

$$
\sigma_{n l}^{b f}=\alpha^{-1} f_{n}\left(\frac{h \nu}{B_{1}}\right) .
$$

Thus, we can write

$$
\alpha_{n l}=\frac{8 \pi(2 l+1)}{\alpha c^{2}}\left(\frac{k_{B} T}{2 \pi m_{e}}\right)^{3 / 2} e^{\xi / n^{2}} \int_{\xi / n^{2}}^{\infty} d y f_{n}\left(\frac{y}{\xi}\right) \frac{y^{2}}{e^{y}-1},
$$

where

$$
\xi=\frac{B_{1}}{k_{B} T}=\frac{m_{e} c^{2} \alpha^{2}}{2 k_{B} T}
$$

and it immediately follows that the $\alpha$ dependence of $\mathcal{R}$ is of the form,

$$
\mathcal{R}=\alpha^{-1} T^{3 / 2} F(\xi)=\alpha^{2} G(\xi) .
$$

As shown in Ref. [32], for the temperatures of interest,

$$
\mathcal{R}=\frac{64}{3} \frac{\hbar^{2} \alpha^{2}}{m_{e}^{2} c} \sqrt{\frac{\pi B_{1}}{3 k_{B} T}} \phi_{2},
$$

where

$$
\phi_{2} \simeq \frac{13 \sqrt{3}}{16 \pi} \ln \left(\frac{B_{1}}{k_{B} T}\right) .
$$

Given the scaling in Eq. (12), we can then read off the $\alpha$ dependence. Explicitly, the recombination coefficient is

$$
\mathcal{R}=\frac{52}{3} \frac{\hbar^{2} \alpha^{3}}{\sqrt{2 \pi m_{e}^{3} k_{B} T}} \ln \left(\frac{m_{e} c^{2} \alpha^{2}}{2 k_{B} T}\right) .
$$

The rate of recombination is inhibited by ionizing photons which can disrupt atoms in the $n=2$ state before they can decay to the ground state. The efficiency of recombination from the $n=2$ state is described by the Peebles efficiency factor,

$$
\mathcal{C}=\frac{\Lambda_{H}+\Lambda_{2 s \rightarrow 1 s}}{\Lambda_{H}+\Lambda_{2 s \rightarrow 1 s}+\beta},
$$

which is just the ratio of the recombination rates to the sum of the recombination and ionization rates from the $n=2$ level.

In Eq. (16),

$$
\Lambda_{H}=\frac{8 \pi H}{\left(\lambda_{2 p \rightarrow 1 s}\right)^{3} n_{1 s}}
$$

is the rate at which a recombination is successful because the emitted Lyman- $\alpha$ photon is redshifted out of the Lyman- $\alpha$ line before ionizing a hydrogen atom. Here $H=(1 / a)$ $\times(d a / d t)$ is the Hubble expansion rate, $n_{1 s} \simeq\left(1-x_{e}\right) n_{p}$ is the number density of atoms in the $1 s$ state (almost all hydrogen atoms are in the $1 s$ state), and 


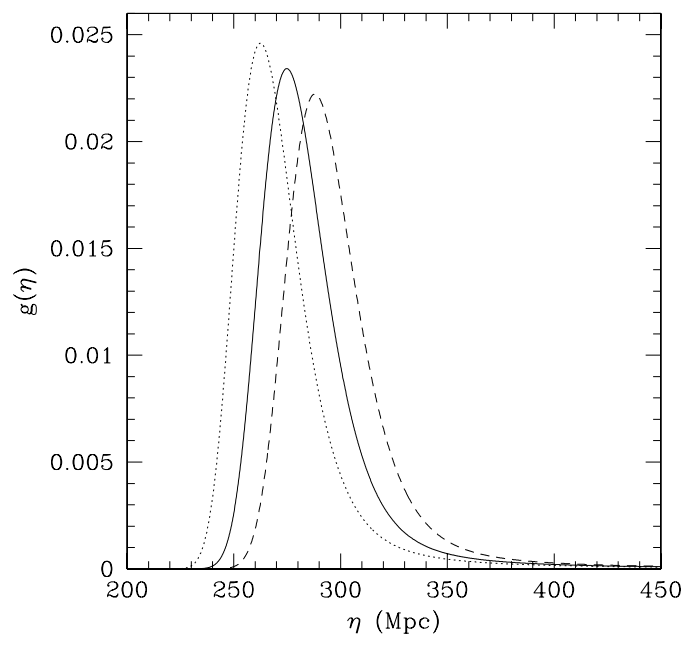

FIG. 1. The probability distribution for the last scattering of a photon, the visibility function, as a function of conformal time $\eta$ in the $\Lambda$ CDM model for $\varphi=\left(\alpha-\alpha_{0}\right) / \alpha_{0}=0.03$ (dotted line), $\varphi=0$ (solid line), and $\varphi=-0.03$ (dashed line).

$$
\lambda_{2 p \rightarrow 1 s}=\frac{16 \pi \hbar}{3 m_{e} c \alpha^{2}},
$$

is the Lyman- $\alpha$ rest wavelength.

The two-photon process $2 s \rightarrow 1 s$ proceeds through virtual atomic states at a rate $\Lambda_{2 s \rightarrow 1 s}=8.22458 \mathrm{~s}^{-1}$ [33], and scales as [34]

$$
\Lambda_{2 s \rightarrow 1 s} \propto \alpha^{8} .
$$

Equations (4)-(19) account for the $\alpha$ dependence of $x_{e}(t)$. Along with the $\alpha$ dependence of $\sigma_{T}$, this completely determines how $g(t)$ varies with $\alpha$ in a given cosmology. While we expect a more complete calculation of recombination, such as that in Ref. [35], may yield further refinements to the $\alpha$ dependence, modifying hydrogen recombination as described above is adequate for our purposes because we are primarily concerned with the $\alpha$ dependence of the visibility function at very high redshift where the simple calculation accurately captures the physics. Also, it was determined in Ref. [28] that other effects, such as modifications to the details of helium recombination, or the cooling of baryons, are small compared to the effect of variations of $\alpha$ on hydrogen recombination.

In this paper we work within the flat geometry $\Lambda \mathrm{CDM}$ cosmology with baryon and matter densities $\Omega_{b}=0.05$ and $\Omega_{m}=0.30$, the Hubble parameter $h=0.72$, and spectral in$\operatorname{dex} n=1$. Figure 1 shows the visibility function $g(\eta)$ $=\exp (-\tau) d \tau / d \eta$ plotted versus the conformal time $\eta$ $=\int d t / a$ for three different values of $\varphi=\left(\alpha-\alpha_{0}\right) / \alpha_{0}$, where $\alpha_{0}=0.00729735 \simeq 1 / 137$ is the value of the electromagnetic fine structure parameter [36]. For positive values of $\varphi$ the visibility function is narrower and peaks earlier, while for negative values of $\varphi$ the visibility function is broader and peaks later. These effects impact directly the CMB angular power spectrum because the peak of the visibility function determines the physical distance to the last scattering sur-

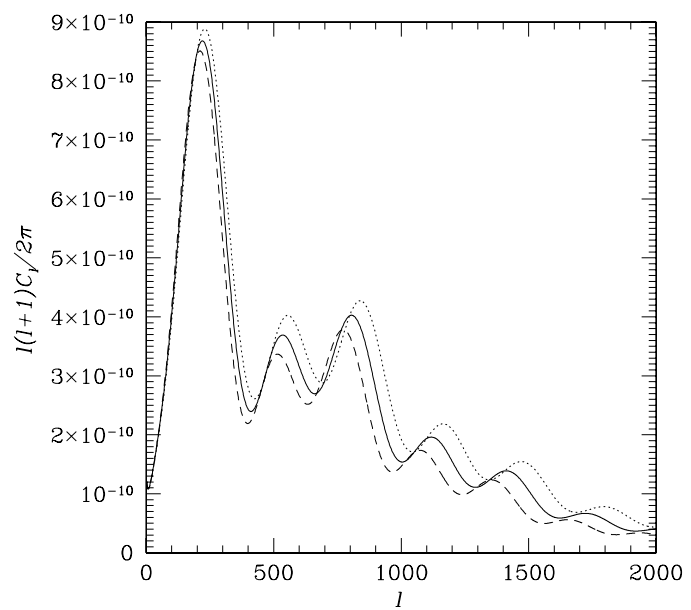

FIG. 2. The angular power spectrum of CMB anisotropies for a spatially uniform $\varphi=0.03$ (dotted line), $\varphi=0$ (solid line), and $\varphi$ $=-0.03$ (dashed line).

face, while the width of the visibility function determines the thickness of the last scattering surface.

Figure 2 shows the angular power spectrum of CMB anisotropies calculated assuming a spatially homogeneous value of $\varphi$. The dependence of the spectrum on $\varphi$ is easy to understand qualitatively. For positive $\varphi$ the angular-diameter distance is larger, so the features are scaled systematically to higher values of $l$. The last-scattering surface is also narrower so that small-scale (high-l) features are less damped due to photon diffusion. For negative $\varphi$ the opposite holds, the smaller angular-diameter distance scales features to lower values of $l$ while the broader last-scattering surface leads to more damping of power on small scales. In the next two sections we derive the changes in the angular power spectrum due to spatial fluctuations in $\varphi$ between causally disconnected regions of the Universe.

\section{POWER SPECTRA}

\section{A. CMB power spectra fundamentals}

The CMB radiation is observed to be a nearly isotropic background of blackbody radiation at a temperature of $T_{\mathrm{CMB}}=2.728 \pm 0.004 \mathrm{~K}$ [37]. Anisotropies in the temperature are observed with a fractional amplitude of $\sim 10^{-5}$ [38], and in the polarization with a fractional amplitude of $\sim 10^{-6}$ [39]. For a review of the physics of CMB anisotropies see, e.g., Ref. [40].

The fundamental CMB anisotropy observables are the Stokes parameters $(\Theta, Q, U, V)$, which can be expressed in the Pauli basis as (see, for example, Ref. [41])

$$
\mathbf{P}(\hat{\mathbf{n}})=\Theta(\hat{\mathbf{n}}) \mathbf{1}+Q(\hat{\mathbf{n}}) \boldsymbol{\sigma}_{3}+U(\hat{\mathbf{n}}) \boldsymbol{\sigma}_{1}+V(\hat{\mathbf{n}}) \boldsymbol{\sigma}_{2}
$$

In this expression

$$
\Theta(\hat{\mathbf{n}})=\frac{T(\hat{\mathbf{n}})-T_{\mathrm{CMB}}}{T_{\mathrm{CMB}}}
$$


denotes the fractional temperature anisotropy in a direction $\hat{\mathbf{n}}$, and the remaining Stokes parameters are normalized to this quantity. Here $Q$ and $U$ describe independent linear polarization states, while $V$ describes circular polarization. Because circular polarization cannot be generated via Thomson scattering, $V=0$ for the $\mathrm{CMB}$.

It is convenient to introduce the quantities,

$$
{ }_{ \pm} A(\hat{\mathbf{n}})=Q(\hat{\mathbf{n}}) \pm i U(\hat{\mathbf{n}})
$$

which have the spin-2 transformation properties,

$$
{ }_{ \pm} A(\hat{\mathbf{n}}) \rightarrow e^{\mp 2 i \phi}{ }_{ \pm} A(\hat{\mathbf{n}}),
$$

under a counterclockwise rotation of the coordinate axis by an angle $\phi$.

We can expand $\Theta(\hat{\mathbf{n}})$ and ${ }_{ \pm} A(\hat{\mathbf{n}})$ in normal modes as [42]

$$
\Theta(\hat{\mathbf{n}})=\sum_{l=1}^{\infty} \sum_{m=-l}^{m=l}(-i)^{l} \sqrt{\frac{4 \pi}{2 l+1}} \Theta_{l m} Y_{l}^{m}(\hat{\mathbf{n}}),
$$

and

$$
{ }_{ \pm} A(\hat{\mathbf{n}})=\sum_{l=1}^{\infty} \sum_{m=-l}^{m=l}(-i)^{l} \sqrt{\frac{4 \pi}{2 l+1}}\left({ }_{ \pm} A_{l m}\right)\left[{ }_{ \pm 2} Y_{l}^{m}(\hat{\mathbf{n}})\right],
$$

where

$$
\Theta_{l m}=\int \frac{d^{3} \mathbf{k}}{(2 \pi)^{3}} \Theta_{l}^{(m)}(k) e^{i \mathbf{k} \cdot \mathbf{x}},
$$

and

$$
{ }_{ \pm} A_{l m}=\int \frac{d^{3} \mathbf{k}}{(2 \pi)^{3}}\left({ }_{ \pm} A_{l}^{(m)}(k)\right) e^{i \mathbf{k} \cdot \mathbf{x}} .
$$

Here ${ }_{s} Y_{l}^{m}$ are the spin-s weighted spherical harmonics [43], with $Y_{l}^{m}={ }_{0} Y_{l}^{m}$, and $X_{l}^{(m)}(k)$ is the contribution to the angular mode $X_{l m}$ from wave vectors of the primordial density field of magnitude $k$.

It is conventional to write the polarization in terms of the moments of the curl-free (scalar) configurations $E_{l m}$, and the moments of the divergence-free (pseudo-scalar) configurations $B_{l m}$, where

$$
{ }_{ \pm} A_{l m}=E_{l m} \pm i B_{l m} .
$$

We can then provide a complete description of an arbitrary CMB anisotropy field using the moments $\Theta_{l m}, E_{l m}$, and $B_{l m}$.

The basic observables of the random fields $X(\hat{\mathbf{n}})$ are the power spectra $C_{l}^{X \tilde{X}}$, defined by

$$
\left\langle X_{l m}^{*} \widetilde{X}_{l^{\prime} m^{\prime}}\right\rangle=\delta_{l l^{\prime}} \delta_{m m^{\prime}} C_{l}^{X \tilde{X}},
$$

where $X, \widetilde{X} \in\{\Theta, E, B\}$ and the angle brackets denote an average over all realizations. Here,

$$
C_{l}^{X \tilde{X}}=\frac{2}{\pi(2 l+1)^{2}} \int \frac{d k}{k} \sum_{m=-2}^{2} k^{3} X_{l}^{(m) *}(k) \widetilde{X}_{l}^{(m)}(k) .
$$

A set of Gaussian random fields-and we expect $\{\Theta, E, B\}$ to be Gaussian-are completely characterized by their power spectra and cross-power spectra. Because the pseudo-scalar $B$ has opposite parity to the scalars $\Theta$ and $E$ the only non-vanishing power spectra are $C_{l}^{\Theta \Theta}, C_{l}^{\Theta E}, C_{l}^{E E}$, and $C_{l}^{B B}$.

For small patches of sky it is an excellent approximation to treat the sky as flat and expand the field $X(\hat{\mathbf{n}})$ in spinweighted Fourier modes rather than spin-weighted spherical harmonics. Thus we have (for example, Ref. [44])

$$
\begin{gathered}
\Theta(\hat{\mathbf{n}})=\int \frac{d^{2} \mathbf{l}}{(2 \pi)^{2}} \Theta(\mathbf{l}) e^{i \mathbf{l} \cdot \hat{\mathbf{n}}}, \\
{ }_{ \pm} A(\hat{\mathbf{n}})=-\int \frac{d^{2} \mathbf{l}}{(2 \pi)^{2}} \pm(\mathbf{l}) e^{ \pm 2 i\left(\phi_{l}-\phi\right)} e^{i \mathbf{l} \cdot \hat{\mathbf{n}}},
\end{gathered}
$$

and we again define $E$ and $B$ through

$$
{ }_{ \pm} A(\mathbf{l})=E(\mathbf{l}) \pm i B(\mathbf{l}) .
$$

In this notation the power spectra are defined by

$$
\left\langle X(\mathbf{l}) \widetilde{X}\left(\mathbf{l}^{\prime}\right)\right\rangle=(2 \pi)^{2} \delta^{2}\left(\mathbf{l}+\mathbf{l}^{\prime}\right) C_{l}^{X \tilde{X}} .
$$

Unless otherwise noted, we work within this flat-sky approximation for the remainder of the paper.

\section{B. Derivative power spectra}

How do the expressions for the power spectra change if we allow for spatial fluctuations of $\alpha$ ? As a warmup we first consider a spatially uniform variation, $\alpha=\alpha_{0}(1+\varphi)$, where $\varphi \ll 1$.

For a given primordial density field $\delta(\mathbf{x})$, the temperature and polarization patterns $\Theta(\hat{\mathbf{n}})$ and ${ }_{ \pm} A(\hat{\mathbf{n}})$ can be calculated by solving the combined Einstein equations and radiativetransfer equations, as well as the equations for the recombination history. As discussed above, this recombination history depends on $\alpha$. Thus, the temperature and polarization fields are implicitly functions of $\varphi=\left(\alpha-\alpha_{0}\right) / \alpha_{0}$. We can expand $\Theta(\hat{\mathbf{n}})=\Theta(\hat{\mathbf{n}} ; \varphi)$ and ${ }_{ \pm} A(\hat{\mathbf{n}})={ }_{ \pm} A(\hat{\mathbf{n}} ; \varphi)$ in Taylor series about $\varphi=0$,

$$
\Theta(\hat{\mathbf{n}})=\Theta_{0}(\hat{\mathbf{n}})+\partial_{\varphi} \Theta_{0}(\hat{\mathbf{n}}) \varphi+\frac{1}{2} \partial_{\varphi}^{2} \Theta_{0}(\hat{\mathbf{n}}) \varphi^{2}+\cdots
$$

${ }_{ \pm} A(\hat{\mathbf{n}})={ }_{ \pm} A_{0}(\hat{\mathbf{n}})+\partial_{\varphi}\left({ }_{ \pm} A_{0}(\hat{\mathbf{n}})\right) \varphi+\frac{1}{2} \partial_{\varphi}^{2}\left({ }_{ \pm} A_{0}(\hat{\mathbf{n}})\right) \varphi^{2}+\cdots$

Inverting Eqs. (31) and (32), we find that 


$$
\Theta(\mathbf{l})=\int d^{2} \hat{\mathbf{n}} \Theta(\hat{\mathbf{n}}) e^{-i \mathbf{l} \cdot \hat{\mathbf{n}}}
$$

and

$$
{ }_{ \pm} A(\mathbf{l})=-\int d^{2} \hat{\mathbf{n}}\left({ }_{ \pm} A(\hat{\mathbf{n}})\right) e^{ \pm 2 i\left(\phi-\phi_{l}\right)} e^{-i \mathbf{l} \cdot \hat{\mathbf{n}}} .
$$

Thus, the $\varphi$ expansions can be written in $\mathbf{l}$ space as

$$
\begin{gathered}
\Theta(\mathbf{l})=\Theta_{0}(\mathbf{l})+\partial_{\varphi} \Theta_{0}(\mathbf{l}) \varphi+\frac{1}{2} \partial_{\varphi}^{2} \Theta_{0}(\mathbf{l}) \varphi^{2}+\cdots, \\
{ }_{ \pm} A(\mathbf{l})={ }_{ \pm} A_{0}(\mathbf{l})+\partial_{\varphi}\left({ }_{ \pm} A_{0}(\mathbf{l})\right) \varphi+\frac{1}{2} \partial_{\varphi}^{2}\left({ }_{ \pm} A_{0}(\mathbf{l})\right) \varphi^{2}+\cdots,
\end{gathered}
$$

and in fact for any field $X \in\{\Theta, E, B\}$ we may write

$$
X(\mathbf{l})=X_{0}(\mathbf{l})+\partial_{\varphi} X_{0}(\mathbf{l}) \varphi+\frac{1}{2} \partial_{\varphi}^{2} X_{0}(\mathbf{l}) \varphi^{2}+\cdots
$$

To $O\left(\varphi^{2}\right)$ we then have

$$
\begin{aligned}
\left\langle X(\mathbf{l}) \widetilde{X}\left(\mathbf{l}^{\prime}\right)\right\rangle= & \left\langle X_{0}(\mathbf{l}) \widetilde{X}_{0}\left(\mathbf{l}^{\prime}\right)\right\rangle+\left\langle X_{0}(\mathbf{l}) \partial_{\varphi} \widetilde{X}_{0}\left(\mathbf{l}^{\prime}\right)\right\rangle \varphi \\
& +\left\langle\partial_{\varphi} X_{0}(\mathbf{l}) \widetilde{X}_{0}\left(\mathbf{l}^{\prime}\right)\right\rangle \varphi+\frac{1}{2}\left\langle X_{0}(\mathbf{l}) \partial_{\varphi}^{2} \widetilde{X}_{0}\left(\mathbf{l}^{\prime}\right)\right\rangle \varphi^{2} \\
& +\frac{1}{2}\left\langle\partial_{\varphi}^{2} X_{0}(\mathbf{l}) \widetilde{X}_{0}\left(\mathbf{l}^{\prime}\right)\right\rangle \varphi^{2} \\
& +\left\langle\partial_{\varphi} X_{0}(\mathbf{l}) \partial_{\varphi} \tilde{X}_{0}\left(\mathbf{l}^{\prime}\right)\right\rangle \varphi^{2}
\end{aligned}
$$

and in terms of power spectra this becomes

$$
\begin{aligned}
C_{l}^{X \tilde{X}}= & C_{l}^{X_{0} \tilde{X}_{0}}+\left(C_{l}^{X_{0} \partial \tilde{X}_{0}}+C_{l}^{\partial X_{0} \tilde{X}_{0}}\right) \varphi \\
& +\left(\frac{1}{2} C_{l}^{X_{0} \partial^{2} \tilde{X}_{0}}+\frac{1}{2} C_{l}^{\partial^{2} X_{0} \tilde{X}_{0}}+C_{l}^{\partial X_{0} \partial \tilde{X}_{0}}\right) \varphi^{2} .
\end{aligned}
$$

Since we are for the time being assuming a spatially uniform value of $\varphi$ we may also write

$$
C_{l}^{X \tilde{X}}=\left.C_{l}^{X \tilde{X}}\right|_{0}+\left.\partial_{\varphi} C_{l}^{X \tilde{X}}\right|_{0} \varphi+\left.\frac{1}{2} \partial_{\varphi}^{2} C_{l}^{X \tilde{X}}\right|_{0} \varphi^{2} .
$$

This allows us to make the identifications

$$
\begin{gathered}
\left.C_{l}^{X \tilde{X}}\right|_{0}=C_{l}^{X \tilde{X}_{0}}, \\
\left.\partial_{\varphi} C_{l}^{X \tilde{X}}\right|_{0}=C_{l}^{X X_{0} \partial \tilde{X}_{0}}+C_{l}^{\partial X_{0} \tilde{X}_{0}}, \\
\left.\partial_{\varphi}^{2} C_{l}^{X \tilde{X}}\right|_{0}=C_{l}^{X} \partial^{2} \tilde{X}_{0}+C_{l}^{\partial^{2} X_{0} \tilde{X}_{0}}+2 C_{l}^{\partial X_{0} \partial \tilde{X}_{0}} .
\end{gathered}
$$

These identifications make it clear how to calculate the individual "derivative" power spectra. We just differentiate Eq.

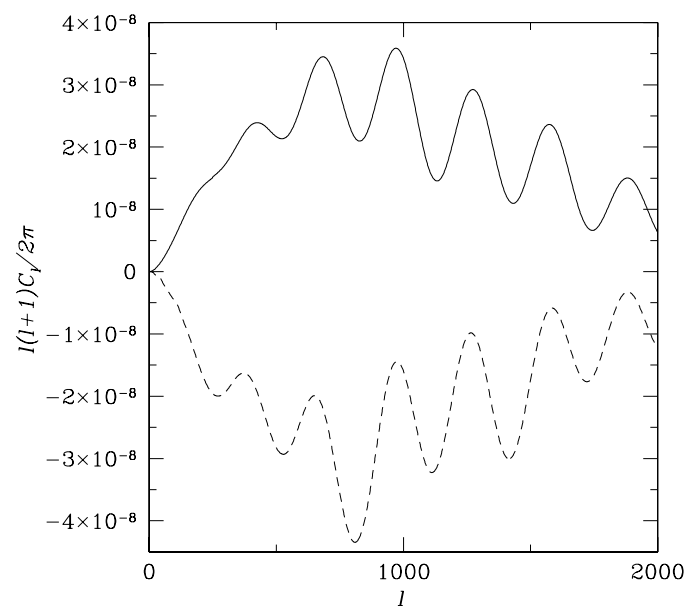

FIG. 3. The $C_{l}^{\partial \Theta_{0} \partial \Theta_{0}}$ (solid line) and $C_{l}^{\Theta_{0} \partial^{2} \Theta_{0}}$ (dashed line) derivative power spectra.

(30) with respect to $\varphi$, evaluate the expression at $\varphi=0$, and pick off the terms with the requisite structure.

For example,

$$
\begin{aligned}
C_{l}^{\partial X_{0} \partial \tilde{X}_{0}=} & \frac{2}{\pi(2 l+1)^{2}} \int \frac{d k}{k} \sum_{m=-2}^{2} k^{3} \\
& \times\left.\partial_{\varphi} X_{l}^{(m) *}(k) \partial_{\varphi} \widetilde{X}_{l}^{(m)}(k)\right|_{\varphi=0},
\end{aligned}
$$

where factors like $\partial_{\varphi} \widetilde{X}_{l}^{(m)}(k)$ can be calculated numerically or directly from first principles using the expressions for the $\widetilde{X}_{l}^{(m)}(k)$ derived in, for example, Ref. [45]. Because they are used in subsequent calculations we have created a modified version of the code CMBFAST [46] that can compute these derivative power spectra. (See Figs. 3-5.)

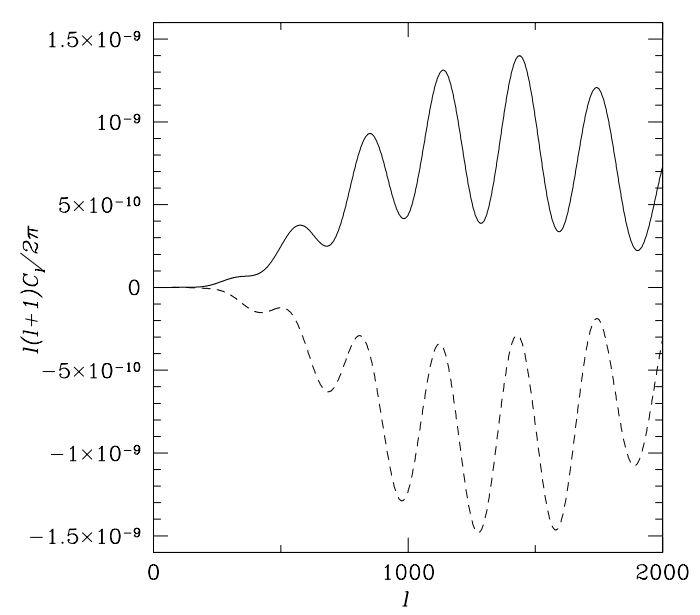

FIG. 4. The $C_{l}^{\partial E_{0} \partial E_{0}}$ (solid line) and $C_{l}^{E_{0} \partial^{2} E_{0}}$ (dashed line) derivative power spectra. 


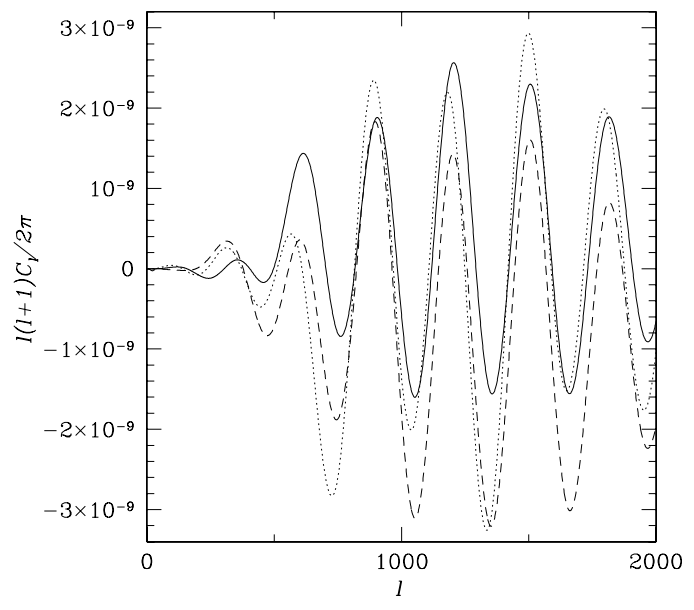

FIG. 5. The $C_{l}^{\partial \Theta_{0} \partial E_{0}}$ (solid line), $C_{l}^{\Theta_{0} \partial^{2} E_{0}}$ (dashed line) and $C_{l}^{E_{0} \partial^{2} \Theta_{0}}$ (dotted line) derivative power spectra.

\section{SPATIAL VARIATIONS OF $\alpha$}

We now consider the effects of spatial variations of $\alpha$ (parametrized by $\varphi$ ) between different causally disconnected regions of the Universe. First suppose that there were no density fluctuations, but spatial variations of $\alpha$. In that case, photons from different points on the sky would be last scattered at different cosmological times, but they would all still have the same frequency when observed by us. However, if there are density fluctuations, the manner in which they are imprinted on the CMB depends on the value of $\alpha$, as discussed above. Thus, if there are spatial variations in $\alpha$, the power spectra (or two-point correlation functions) will vary from one place on the sky to another. This implies that the stochasticity of the spatial variations in $\alpha$ induce nonGaussianity in the CMB quantified by non-zero (connected) higher order correlation functions (trispectra and perhaps bispectra). It also implies a correction to the mean power spectrum (i.e., that measured by mapping regions of the sky that contain many coherence regions of $\alpha$ ), as well as the introduction of a non-zero curl in the polarization. All of these effects are analogous to similar effects induced by weak lensing of the CMB. The only difference is that in our case, the temperature and polarization patterns are modulated by a variable $\alpha$, rather than lensing by an intervening density field along the line of sight.

In this section, we first calculate the modified power spec$\operatorname{tra} C_{l}^{\Theta \Theta}, C_{l}^{\Theta E}, C_{l}^{E E}$, and $C_{l}^{B B}$. We then determine the form of the higher order correlations (bispectra and trispectra) in the next two sections.

\section{A. Observable modes in the presence of $\varphi$ fluctuations}

We assume that at a given position $\hat{\mathbf{n}}$ at the surface of last scatter, the value of $\alpha$ is $\alpha(\hat{\mathbf{n}})=\alpha_{0}[1+\varphi(\hat{\mathbf{n}})]$. Here we treat $\varphi(\hat{\mathbf{n}})$ as a random field with angular power spectrum $\left\langle\varphi(\mathbf{I}) \varphi\left(\mathbf{I}^{\prime}\right)\right\rangle=(2 \pi)^{2} \delta^{2}\left(\mathbf{I}+\mathbf{I}^{\prime}\right) C_{l}^{\varphi \varphi}$ in the flat-sky approximation.

We assume that the surface of last scatter is much thinner than the spatial correlation length of $\varphi$, and that in a given direction $\alpha$ is constant throughout recombination. We also assume that the dynamics responsible for the variations of $\varphi$ have a negligible effect on the perturbation evolution so that the sole effect of variations of $\varphi$ are a modification of the microphysics. We will discuss the validity of these assumptions later.

Again we expand our fields $\Theta(\hat{\mathbf{n}})=\Theta(\hat{\mathbf{n}} ; \varphi)$ and ${ }_{ \pm} A(\hat{\mathbf{n}})$ $={ }_{ \pm} A(\hat{\mathbf{n}} ; \varphi)$ in a Taylor series about $\varphi=0$ as

$$
\begin{aligned}
\Theta(\hat{\mathbf{n}})= & \Theta_{0}(\hat{\mathbf{n}})+\partial_{\varphi} \Theta_{0}(\hat{\mathbf{n}}) \varphi(\hat{\mathbf{n}})+\frac{1}{2} \partial_{\varphi}^{2} \Theta_{0}(\hat{\mathbf{n}}) \varphi^{2}(\hat{\mathbf{n}}), \\
{ }_{ \pm} A(\hat{\mathbf{n}})= & { }_{ \pm} A_{0}(\hat{\mathbf{n}})+\partial_{\varphi}\left({ }_{ \pm} A_{0}(\hat{\mathbf{n}})\right) \varphi(\hat{\mathbf{n}}) \\
& +\frac{1}{2} \partial_{\varphi}^{2}\left({ }_{ \pm} A_{0}(\hat{\mathbf{n}})\right) \varphi^{2}(\hat{\mathbf{n}}),
\end{aligned}
$$

where $\varphi(\hat{\mathbf{n}})$ is now a function of position.

By taking the Fourier transform of $\Theta(\hat{\mathbf{n}})$ we find

$$
\Theta(\mathbf{l})=\Theta_{0}(\mathbf{l})+\left[\left(\partial_{\varphi} \Theta_{0}\right) \star \varphi\right](\mathbf{l})+\frac{1}{2}\left[\left(\partial_{\varphi}^{2} \Theta_{0}\right) \star \varphi \star \varphi\right](\mathbf{l}),
$$

where

$$
[X \star \psi](\mathbf{I})=\int \frac{d^{2} \mathbf{l}^{\prime}}{(2 \pi)^{2}} X\left(\mathbf{l}^{\prime}\right) \psi\left(\mathbf{l}-\mathbf{l}^{\prime}\right)
$$

is the convolution of two fields $X$ and $\psi$, and

$$
[X \star \psi \star \lambda](\mathbf{l})=\int \frac{d^{2} \mathbf{l}^{\prime}}{(2 \pi)^{2}}[X \star \psi]\left(\mathbf{l}^{\prime}\right) \lambda\left(\mathbf{l}-\mathbf{l}^{\prime}\right)
$$

is the double convolution of three fields $X, \psi$, and $\lambda$.

Similarly, taking linear combinations of the Fourier transforms of ${ }_{ \pm} A(\hat{\mathbf{n}})$ we find that

$$
\begin{aligned}
E(\mathbf{l})= & E_{0}(\mathbf{l})+\left[\left(\partial_{\varphi} E_{0}\right) \star_{\mathbf{c}} \varphi\right](\mathbf{l})-\left[\left(\partial_{\varphi} B_{0}\right) \star_{\mathbf{S}} \varphi\right](\mathbf{l}) \\
& +\frac{1}{2}\left[\left(\partial_{\varphi}^{2} E_{0}\right) \star_{c} \varphi \star \varphi\right](\mathbf{l})-\frac{1}{2}\left[\left(\partial_{\varphi}^{2} B_{0}\right) \star_{\mathbf{S}} \varphi \star_{\varphi}\right](\mathbf{l}),
\end{aligned}
$$

and

$$
\begin{aligned}
B(\mathbf{l})= & B_{0}(\mathbf{l})+\left[\left(\partial_{\varphi} B_{0}\right) \star_{\mathbf{c}} \varphi\right](\mathbf{l})+\left[\left(\partial_{\varphi} E_{0}\right) \star_{\mathbf{S}} \varphi\right](\mathbf{l}) \\
& +\frac{1}{2}\left[\left(\partial_{\varphi}^{2} B_{0}\right) \star_{c} \varphi \star_{\varphi}\right](\mathbf{l})+\frac{1}{2}\left[\left(\partial_{\varphi}^{2} E_{0}\right) \star_{\mathbf{S}} \varphi \star_{\varphi}\right](\mathbf{l}),
\end{aligned}
$$

where

$$
\left[X \star_{\mathbf{c}} \psi\right](\mathbf{l})=\int \frac{d^{2} \mathbf{l}^{\prime}}{(2 \pi)^{2}} \cos \left(2 \phi_{l^{\prime}}\right) X\left(\mathbf{l}^{\prime}\right) \psi\left(\mathbf{l}-\mathbf{l}^{\prime}\right),
$$


and

$$
\left[X \star_{\mathbf{s}} \psi\right](\mathbf{l})=\int \frac{d^{2} \mathbf{l}^{\prime}}{(2 \pi)^{2}} \sin \left(2 \phi_{l^{\prime}}\right) X\left(\mathbf{l}^{\prime}\right) \psi\left(\mathbf{l}-\mathbf{l}^{\prime}\right),
$$

are the even- and odd-parity spin-2 weighted convolutions of $X$ and $\psi$, respectively.

Examining these expressions we find that a given mode $X$ (I) receives corrections due to the combination of modes $\left\{\partial_{\varphi}^{n} X_{0}\left(\mathbf{l}_{0}\right), \varphi\left(\mathbf{l}_{1}\right), \varphi\left(\mathbf{l}_{2}\right), \ldots, \varphi\left(\mathbf{l}_{n}\right)\right\}$ such that $\sum_{i=0}^{n} \mathbf{l}_{i}=\mathbf{l}$. Furthermore, the $E$ and $B$ modes mix so that, for example, the mode $B(\mathbf{l})$ can be induced by the combinations of modes $\left\{\partial_{\varphi}^{n} E_{0}\left(\mathbf{l}_{0}\right), \varphi\left(\mathbf{l}_{1}\right), \varphi\left(\mathbf{l}_{2}\right), \ldots, \varphi\left(\mathbf{l}_{n}\right)\right\}$ such that $\sum_{i=0}^{n} \mathbf{l}_{i}=\mathbf{l}$. These effects modify the angular power spectra of $\mathrm{CMB}$ anisotropies, and introduce higher-order connected (nonGaussian) correlation functions.

\section{B. The $\Theta \Theta$ power spectrum}

Using Eq. (49) we find that the expansion for the two point correlation function (in Fourier space) is

$$
\begin{aligned}
\left\langle\Theta(\mathbf{l}) \Theta\left(\mathbf{l}^{\prime}\right)\right\rangle= & \left\langle\Theta_{0}(\mathbf{l}) \Theta_{0}\left(\mathbf{l}^{\prime}\right)\right\rangle+\left\langle\Theta_{0}(\mathbf{l})\left[\partial_{\varphi} \Theta_{0} \star \varphi\right]\left(\mathbf{l}^{\prime}\right)\right\rangle \\
& +\left\langle\left[\partial_{\varphi} \Theta_{0} \star \varphi\right](\mathbf{l}) \Theta_{0}\left(\mathbf{l}^{\prime}\right)\right\rangle \\
& +\frac{1}{2}\left\langle\Theta_{0}(\mathbf{l})\left[\partial_{\varphi}^{2} \Theta_{0} \star \varphi \star \varphi\right]\left(\mathbf{l}^{\prime}\right)\right\rangle \\
& +\frac{1}{2}\left\langle\left[\partial_{\varphi}^{2} \Theta_{0} \star \varphi \star \varphi\right](\mathbf{l}) \Theta_{0}\left(\mathbf{l}^{\prime}\right)\right\rangle \\
& +\left\langle\left[\partial_{\varphi} \Theta_{0} \star \varphi\right](\mathbf{l})\left[\partial_{\varphi} \Theta_{0} \star \varphi\right]\left(\mathbf{l}^{\prime}\right)\right\rangle
\end{aligned}
$$

to $O\left(\varphi^{2}\right)$. In the above expression and what follows we adopt the convention that the differential operators $\partial_{\varphi}$ act only the field immediately following them.

We assume that $\Theta_{0}$ and $\varphi$ are zero-mean Gaussian random fields without higher-order connected correlators. By writing out the convolutions and Wick expanding the correlators it is easy to verify that correlators involving an odd number of fields vanish, and so there are no corrections to first order in $\varphi$. It is also straightforward to verify that

$$
\begin{aligned}
\left\langle\Theta_{0}(\mathbf{l})\right. & {\left.\left[\partial_{\varphi}^{2} \Theta_{0} \star \varphi \star \varphi\right]\left(\mathbf{l}^{\prime}\right)\right\rangle } \\
= & \left\langle\left[\partial_{\varphi}^{2} \Theta_{0} \star \varphi \star \varphi\right](\mathbf{l}) \Theta_{0}\left(\mathbf{l}^{\prime}\right)\right\rangle \\
= & (2 \pi)^{2} \delta^{2}\left(\mathbf{l}+\mathbf{l}^{\prime}\right)\left[\sigma^{(\varphi \varphi)} C_{l}^{\Theta_{0} \partial^{2} \Theta_{0}}+2 \sigma^{\left(\varphi \partial^{2} \Theta_{0}\right)} C_{l}^{\Theta_{0} \varphi}\right],
\end{aligned}
$$

where

$$
\sigma^{(\psi \lambda)}=\int \frac{d^{2} \mathbf{I}}{(2 \pi)^{2}} C_{l}^{\psi \lambda}
$$

is the covariance between two fields $\psi$ and $\lambda$. Similarly we can show that

$$
\begin{aligned}
& \left\langle\left[\partial_{\varphi} \Theta_{0} \star \varphi\right](\mathbf{l})\left[\partial_{\varphi} \Theta_{0} \star \varphi\right]\left(\mathbf{l}^{\prime}\right)\right\rangle \\
& \quad=(2 \pi)^{2} \delta^{2}\left(\mathbf{l}+\mathbf{l}^{\prime}\right)\left\{\left[C^{\varphi \varphi} \star C^{\partial \Theta_{0} \partial \Theta_{0}}\right]_{l}+\left[C^{\partial \Theta_{0} \varphi} \star C^{\partial \Theta_{0} \varphi}\right]_{l}\right\},
\end{aligned}
$$

where we have dropped terms that contribute only when $\mathbf{l}$ $=0$.

Collecting all terms we find that to leading order the average power spectrum, including fluctuations in $\varphi$, is

$$
\begin{aligned}
C_{l}^{\Theta \Theta}= & C_{l}^{\Theta_{0} \Theta_{0}}+\sigma^{(\varphi \varphi)} C_{l}^{\Theta_{0} \partial^{2} \Theta_{0}}+2 \sigma^{\left(\varphi \partial^{2} \Theta_{0}\right)} C_{l}^{\Theta_{0} \varphi} \\
& +\left[C^{\varphi \varphi} \star C^{\partial \Theta_{0} \partial \Theta_{0}}\right]_{l}+\left[C^{\partial \Theta_{0} \varphi} \star C^{\partial \Theta_{0} \varphi}\right]_{l} .
\end{aligned}
$$

Note that the corrections to $C_{l}^{\Theta_{0} \Theta_{0}}$ involve couplings between the derivative power spectra, which are calculated as described in Sec. III B, and $C_{l}^{\varphi \varphi}$ and the various cross power spectra, which are specified by the model that generates spatial variations in $\varphi$.

In general $\Theta_{0}$ and $\varphi$ may be correlated if, for instance, they are generated by a common mechanism or if they are strongly coupled through evolution equations. We discuss in Sec. VII why we do not expect the latter source of correlations to be important as long as the energy in the $\varphi$ field and its fluctuations are small compared to the dominant radiation and matter perturbations. In the case where $\Theta_{0}$ and $\varphi$ have no cross-correlation, the expression simplifies to

$$
C_{l}^{\Theta \Theta}=C_{l}^{\Theta_{0} \Theta_{0}}+\sigma^{(\varphi \varphi)} C_{l}^{\Theta_{0} \partial^{2} \Theta_{0}}+\left[C^{\varphi \varphi} \star C^{\partial \Theta_{0} \partial \Theta_{0}}\right]_{l} .
$$

The result for $C_{l}^{\Theta \Theta}$ will of course depend on $C_{l}^{\varphi \varphi}$. To illustrate we consider a simple model in which $\varphi$ is highly correlated on angular scales smaller than the correlation angle $\theta_{c}$, and uncorrelated on larger scales. (Below we discuss a physical model that may produce such a correlation function.) We thus have

$$
\langle\varphi(0) \varphi(\theta)\rangle=\sigma^{(\varphi \varphi)} e^{-\left(\theta / \theta_{c}\right)^{2}}
$$

In the flat-sky approximation we have

$$
C_{l}^{\varphi \varphi}=\int d^{2} \theta\langle\varphi(0) \varphi(\theta)\rangle e^{-i \mathbf{l} \cdot \boldsymbol{\theta}}
$$

which implies that

$$
C_{l}^{\varphi \varphi}=\pi \theta_{c}^{2} \sigma^{(\varphi \varphi)} e^{-(1 / 4) l^{2} \theta_{c}^{2}}
$$

In this case the average $\Theta \Theta$ power spectrum is

$$
C_{l}^{\Theta \Theta}=C_{l}^{\Theta_{0} \Theta_{0}}+\sigma^{(\varphi \varphi)}\left[C_{l}^{\Theta_{0} \partial^{2} \Theta_{0}}+\frac{\theta_{c}^{2}}{2} \int d l^{\prime} l^{\prime} e^{-(1 / 4)\left(l^{2}+l^{\prime 2}\right) \theta_{c}^{2}}\right.
$$

$$
\left.\times I_{0}\left(\frac{\theta_{c}^{2}}{2} l l^{\prime}\right) C_{l^{\prime}}^{\partial \Theta_{0} \partial \Theta_{0}}\right]
$$

where $I_{n}$ is the $n$ th-order modified Bessel function of the first kind. We show this average power spectrum in Fig. 6. The main effect of $\varphi$ fluctuations is to reduce the amplitude of oscillatory features in the damping tail. This effect can be 


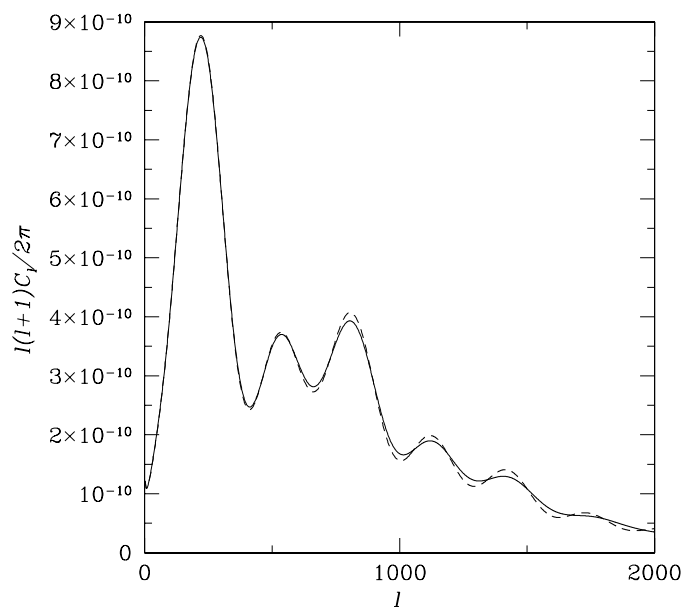

FIG. 6. The solid curve shows the $\Theta \Theta$ power spectrum for the Gaussian correlation function for $\varphi$ with angular correlation scale $\theta_{c}=1^{\circ}$ and variance $\sigma^{(\varphi \varphi)}=9 \times 10^{-4}$ (fluctuations in $\varphi$ at the $3 \%$ level). The dashed curve shows the power spectrum without fluctuations in $\varphi$.

understood by noting that patches of the sky with different values of $\varphi$ will have different power spectra, and that, as can be seen in Fig. 2, the location of the peaks in the damping tail of these power spectra shift as $\varphi$ changes. These patch power spectra add incoherently, so that the amplitude of the oscillatory component of the average power spectrum is reduced. This is the same type of effect as in weak gravitational lensing [47]. As we will see, this is the predominant effect in the other power spectra as well.

\section{The $\Theta E$ power spectrum}

Using Eqs. (49) and (52), and immediately dropping the vanishing correlators involving an odd number of fields, we find that the expansion for the power spectrum is

$$
\begin{aligned}
\left\langle\Theta(\mathbf{I}) E\left(\mathbf{I}^{\prime}\right)\right\rangle= & \left\langle\Theta_{0}(\mathbf{I}) E_{0}\left(\mathbf{I}^{\prime}\right)\right\rangle+\frac{1}{2}\left\langle\Theta_{0}(\mathbf{I})\left[\partial_{\varphi}^{2} E_{0} \star_{\mathbf{c}} \varphi \star \varphi\right]\left(\mathbf{I}^{\prime}\right)\right\rangle \\
& -\frac{1}{2}\left\langle\Theta_{0}(\mathbf{I})\left[\partial_{\varphi}^{2} B_{0} \star_{\mathbf{S}} \varphi \star \varphi\right]\left(\mathbf{I}^{\prime}\right)\right\rangle \\
& +\left\langle\left[\partial_{\varphi} \Theta_{0} \star \varphi\right](\mathbf{l})\left[\partial_{\varphi} E_{0} \star_{\mathbf{c}} \varphi\right]\left(\mathbf{I}^{\prime}\right)\right\rangle \\
& -\left\langle\left[\partial_{\varphi} \Theta_{0} \star \varphi\right](\mathbf{l})\left[\partial_{\varphi} B_{0} \star_{\mathbf{S}} \varphi\right]\left(\mathbf{I}^{\prime}\right)\right\rangle \\
& +\frac{1}{2}\left\langle\left[\partial_{\varphi}^{2} \Theta_{0} \star \varphi \star \varphi\right](\mathbf{I}) E_{0}\left(\mathbf{I}^{\prime}\right)\right\rangle
\end{aligned}
$$

to $O\left(\varphi^{2}\right)$.

By writing out the convolutions, Wick expanding the correlators, and noting that terms involving $B_{0}$ vanish due to parity, we find that the non-vanishing terms are

$$
\begin{aligned}
&\left\langle\Theta_{0}(\mathbf{I})\left[\partial_{\varphi}^{2} E_{0} \star \varphi \star \varphi\right]\left(\mathbf{I}^{\prime}\right)\right\rangle \\
&=(2 \pi)^{2} \delta^{2}\left(\mathbf{I}+\mathbf{I}^{\prime}\right)\left[\sigma^{(\varphi \varphi)} C_{l}^{\Theta_{0} \partial^{2} E_{0}}+2 \sigma^{\left(\varphi \partial^{2} E_{0}\right)} C_{l}^{\Theta_{0} \varphi}\right],
\end{aligned}
$$

$$
\begin{gathered}
\left\langle\left[\partial_{\varphi}^{2} \Theta_{0} \star \varphi \star \varphi\right](\mathbf{l}) E_{0}\left(\mathbf{l}^{\prime}\right)\right\rangle= \\
+2 \pi)^{2} \delta^{2}\left(\mathbf{l}+\mathbf{l}^{\prime}\right)\left[\sigma^{(\varphi \varphi)} C_{l}^{E_{0} \partial^{2} \Theta_{0}}\right. \\
\left.+2 \sigma^{\left(\varphi \partial^{2} \Theta_{0}\right)} C_{l}^{E_{0} \varphi}\right] \\
\left\langle\left[\partial_{\varphi} \Theta_{0} \star \varphi\right](\mathbf{l})\left[\partial_{\varphi} E_{0} \star_{\mathbf{c}} \varphi\right]\left(\mathbf{l}^{\prime}\right)\right\rangle \\
=(2 \pi)^{2} \delta^{2}\left(\mathbf{l}+\mathbf{l}^{\prime}\right)\left\{\left[C^{\partial \Theta_{0} \partial E_{0} \star_{\mathbf{c}} C^{\varphi \varphi}}\right]_{l}\right. \\
+\left[C^{\left.\left.\partial E_{0} \varphi \star_{\mathbf{c}} C^{\partial \Theta_{0} \varphi}\right]_{l}\right\}}\right.
\end{gathered}
$$

Collecting these terms we find that, to leading order, the average cross-power spectrum, including fluctuations in $\varphi$, is

$$
\begin{aligned}
C_{l}^{\Theta E}= & C_{l}^{\Theta_{0} E_{0}}+\sigma^{(\varphi \varphi)}\left[\frac{1}{2} C_{l}^{\Theta_{0} \partial^{2} E_{0}}+\frac{1}{2} C_{l}^{E_{0} \partial^{2} \Theta_{0}}\right]+\sigma^{\left(\varphi \partial^{2} E_{0}\right)} C_{l}^{\Theta_{0} \varphi} \\
& +\sigma^{\left(\varphi \partial^{2} \Theta_{0}\right)} C_{l}^{E_{0} \varphi}+\left[C^{\partial \Theta_{0} \partial E_{0} \star_{\mathbf{c}} C^{\varphi \varphi}}\right]_{l} \\
& +\left[C^{\partial E_{0} \varphi} \star_{\mathbf{c}} C^{\partial \Theta_{0} \varphi}\right]_{l} .
\end{aligned}
$$

If $\varphi$ has no correlation with the density field, the expression simplifies to

$$
\begin{aligned}
C_{l}^{\Theta E}= & C_{l}^{\Theta_{0} E_{0}}+\sigma^{(\varphi \varphi)}\left[\frac{1}{2} C_{l}^{\Theta_{0} \partial^{2} E_{0}}+\frac{1}{2} C_{l}^{E_{0} \partial^{2} \Theta_{0}}\right] \\
& +\left[C^{\partial \Theta_{0} \partial E_{0} \star_{\mathbf{c}}} C^{\varphi \varphi}\right]_{l} .
\end{aligned}
$$

Inserting the power spectrum from Eq. (64), we obtain the expression

$$
\begin{aligned}
& C_{l}^{\Theta E}=C_{l}^{\Theta_{0} E_{0}}+\sigma^{(\varphi \varphi)}\left[\frac{1}{2} C_{l}^{\Theta_{0} \partial^{2} E_{0}}+\frac{1}{2} C_{l}^{E_{0} \partial^{2} \Theta_{0}}\right.
\end{aligned}
$$

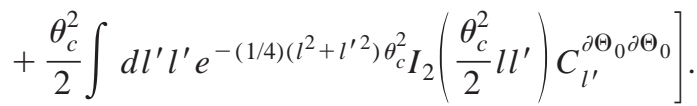

We show this average power spectrum in Fig. 7. The major effect of $\varphi$ fluctuations on the $\Theta E$ power spectrum is to reduce the peak amplitudes on small scales.

\section{The $E E$ power spectrum}

Using Eq. (52), and dropping the correlators involving an odd number of fields and those that vanish due to parity, we find that the expansion for the power spectrum is

$$
\begin{aligned}
\left\langle E(\mathbf{l}) E\left(\mathbf{I}^{\prime}\right)\right\rangle= & \left\langle E_{0}(\mathbf{l}) E_{0}\left(\mathbf{I}^{\prime}\right)\right\rangle+\frac{1}{2}\left\langle E_{0}(\mathbf{l})\left[\partial_{\varphi}^{2} E_{0} \star_{\mathbf{c}} \varphi \star \varphi\right]\left(\mathbf{I}^{\prime}\right)\right\rangle \\
& +\frac{1}{2}\left\langle\left[\partial_{\varphi}^{2} E_{0} \star_{\mathbf{c}} \varphi \star \varphi\right](\mathbf{I}) E_{0}\left(\mathbf{I}^{\prime}\right)\right\rangle \\
& +\left\langle\left[\partial_{\varphi} E_{0} \star_{\mathbf{c}} \varphi\right](\mathbf{l})\left[\partial_{\varphi} E_{0} \star_{\mathbf{c}} \varphi\right]\left(\mathbf{I}^{\prime}\right)\right\rangle \\
& +\left\langle\left[\partial_{\varphi} B_{0} \star_{\mathbf{s}} \varphi\right](\mathbf{l})\left[\partial_{\varphi} B_{0} \star_{\mathbf{s}} \varphi\right]\left(\mathbf{I}^{\prime}\right)\right\rangle
\end{aligned}
$$

to $O\left(\varphi^{2}\right)$. 


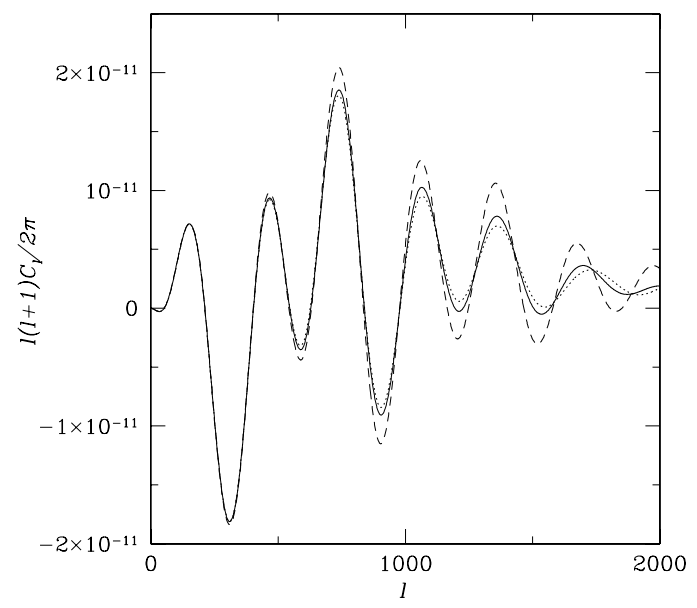

FIG. 7. The $\Theta E$ power spectra for the Gaussian correlation function for $\varphi$ with $\sigma^{(\varphi \varphi)}=9 \times 10^{-4}$ and $\theta_{c}=1^{\circ}$ (solid line) and $\theta_{c}=2^{\circ}$ (dotted line). The dashed line shows the power spectrum without fluctuations in $\varphi$.

After evaluating the convolutions and Wick expanding these correlators we find

$$
\begin{aligned}
\left\langle E_{0}(\mathbf{l})\right. & {\left.\left[\partial_{\varphi}^{2} E_{0} \star_{\mathbf{c}} \varphi \star \varphi\right]\left(\mathbf{I}^{\prime}\right)\right\rangle } \\
= & \left\langle\left[\partial_{\varphi}^{2} E_{0} \star_{c} \varphi \star \varphi\right]\left(\mathbf{I}^{\prime}\right) E_{0}(\mathbf{l})\right\rangle \\
& =(2 \pi)^{2} \delta^{2}\left(\mathbf{l}+\mathbf{l}^{\prime}\right)\left[\sigma^{(\varphi \varphi)} C_{l}^{E_{0} \partial^{2} E_{0}}+2 \sigma^{\left(\varphi \partial^{2} E_{0}\right)} C_{l}^{E_{0} \varphi}\right],
\end{aligned}
$$

$$
\begin{aligned}
\left\langle\left[\partial_{\varphi} E_{0} \star_{\mathbf{c}} \varphi\right](\mathbf{I})\left[\partial_{\varphi} E_{0} \star_{\mathbf{c}} \varphi\right]\left(\mathbf{l}^{\prime}\right)\right\rangle \\
=(2 \pi)^{2} \delta^{2}\left(\mathbf{I}+\mathbf{l}^{\prime}\right) \int \frac{d^{2} \mathbf{l}_{1}}{(2 \pi)^{2}} \\
\quad \times\left\{\cos ^{2}\left(2 \phi_{l_{1}}\right) C_{l_{1}}^{\partial E_{0} \partial E_{0}} C_{\left|\mathbf{I}-\mathbf{l}_{1}\right|}^{\varphi \varphi}+\cos \left(2 \phi_{l_{1}}\right)\right. \\
\left.\times\left[\frac{2 l_{1}^{2} \sin ^{2}\left(\phi_{l_{1}}\right)}{\left|\mathbf{I}-\mathbf{l}_{1}\right|^{2}}-1\right] C_{l_{1}}^{\partial E_{0} \varphi} C_{\left|\mathbf{I}-\mathbf{l}_{1}\right|}^{\partial E_{0} \varphi}\right\},
\end{aligned}
$$

$$
\begin{aligned}
& \left\langle\left[\partial_{\varphi} B_{0} \star_{\mathbf{S}} \varphi\right](\mathbf{I})\left[\partial_{\varphi} B_{0} \star_{\mathbf{S}} \varphi\right]\left(\mathbf{I}^{\prime}\right)\right\rangle \\
& \quad=(2 \pi)^{2} \delta^{2}\left(\mathbf{I}+\mathbf{I}^{\prime}\right) \int \frac{d^{2} \mathbf{l}_{1}}{(2 \pi)^{2}} \sin ^{2}\left(2 \phi_{l_{1}}\right) C_{l_{1}}^{\partial B_{0} \partial B_{0}} C_{\left|\mathbf{l}-\mathbf{I}_{1}\right|}^{\varphi \varphi}
\end{aligned}
$$

Collecting these terms we find that

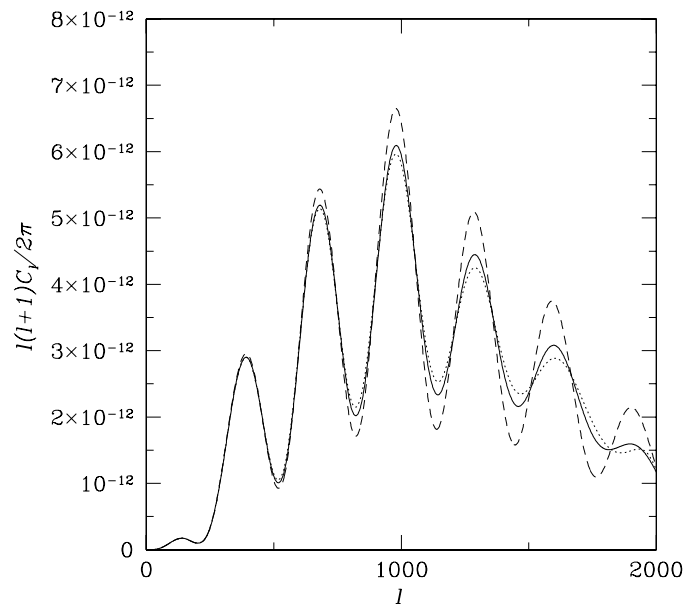

FIG. 8. The $E E$ power spectra for the Gaussian correlation function for $\varphi$ with $\sigma^{(\varphi \varphi)}=9 \times 10^{-4}$ and $\theta_{c}=1^{\circ}$ (solid line) and $\theta_{c}$ $=2^{\circ}$ (dotted line). The dashed line shows the power spectrum without fluctuations in $\varphi$.

$$
\begin{aligned}
C_{l}^{E E}= & C_{l}^{E_{0} E_{0}}+\sigma^{(\varphi \varphi)} C_{l}^{E_{0} \partial^{2} E_{0}}+2 \sigma^{\left(\varphi \partial^{2} E_{0}\right)} C_{l}^{E_{0} \varphi} \\
& +\int \frac{d^{2} \mathbf{l}^{\prime}}{(2 \pi)^{2}}\left\{\left[\cos ^{2}\left(2 \phi_{l^{\prime}}\right) C_{l^{\prime}}^{\partial E_{0} \partial E_{0}}\right.\right. \\
& \left.+\sin ^{2}\left(2 \phi_{l^{\prime}}\right) C_{l^{\prime}}^{\partial B_{0} \partial B_{0}}\right] C_{\left|\mathbf{I}-\mathbf{l}^{\prime}\right|}^{\varphi \varphi}+\cos \left(2 \phi_{l^{\prime}}\right) \\
& \left.\times\left[\frac{2 l^{\prime 2} \sin ^{2}\left(\phi_{l^{\prime}}\right)}{\left|\mathbf{I}-\mathbf{l}^{\prime}\right|^{2}}-1\right] C_{l^{\prime}}^{\partial E_{0} \varphi} C_{\left|\mathbf{I}-\mathbf{I}^{\prime}\right|}^{\partial E_{0} \varphi}\right\}
\end{aligned}
$$

is the expression for the average $E$-mode power spectrum, including fluctuations in $\varphi$.

If $E_{0}$ and $\varphi$ are uncorrelated, the expression simplifies to

$$
\begin{aligned}
C_{l}^{E E}= & C_{l}^{E_{0} E_{0}}+\sigma^{(\varphi \varphi)} C_{l}^{E_{0} \partial^{2} E_{0}}+\int \frac{d^{2} \mathbf{l}^{\prime}}{(2 \pi)^{2}}\left[\cos ^{2}\left(2 \phi_{l^{\prime}}\right) C_{l^{\prime}}^{\partial E_{0} \partial E_{0}}\right. \\
& \left.+\sin ^{2}\left(2 \phi_{l^{\prime}}\right) C_{l^{\prime}}^{\partial B_{0} \partial B_{0}}\right] C_{\left|\mathbf{I}^{-} \mathbf{I}^{\prime}\right|}^{\varphi \varphi}
\end{aligned}
$$

For the $\varphi$ power spectrum in Eq. (64), dropping the negligible primordial $B$-mode term, we obtain the expression

$$
\begin{aligned}
C_{l}^{E E}= & C_{l}^{E_{0} E_{0}}+\sigma^{(\varphi \varphi)}\left\{C_{l}^{E_{0} \partial^{2} E_{0}}\right. \\
& +\frac{\theta_{c}^{2}}{4} \int d l^{\prime} l^{\prime} e^{-(1 / 4)\left(l^{2}+l^{\prime 2}\right) \theta_{c}^{2}}\left[I_{0}\left(\frac{\theta_{c}^{2}}{2} l l^{\prime}\right)\right. \\
& \left.\left.+I_{4}\left(\frac{\theta_{c}^{2}}{2} l l^{\prime}\right)\right] C_{l^{\prime}}^{\partial E_{0} \partial E_{0}}\right\} .
\end{aligned}
$$

We plot this average power spectrum in Fig. 8. Again, the 
effect of $\varphi$ fluctuations is to reduce the amplitude of the oscillatory component at small angular scales.

\section{E. The $B B$ power spectrum}

Using Eq. (53), and once again dropping the correlators involving an odd number of fields and those that vanish due to parity, we find that the expansion for the two-point correlation function is

$$
\begin{aligned}
\left\langle B(\mathbf{l}) B\left(\mathbf{I}^{\prime}\right)\right\rangle= & \left\langle B_{0}(\mathbf{l}) B_{0}\left(\mathbf{l}^{\prime}\right)\right\rangle+\frac{1}{2}\left\langle B_{0}(\mathbf{l})\left[\partial_{\varphi}^{2} B_{0} \star_{\mathbf{c}} \varphi \star \varphi\right]\left(\mathbf{l}^{\prime}\right)\right\rangle \\
& +\frac{1}{2}\left\langle\left[\partial_{\varphi}^{2} B_{0} \star_{\mathbf{c}} \varphi \star \varphi\right](\mathbf{l}) B_{0}\left(\mathbf{l}^{\prime}\right)\right\rangle \\
& +\left\langle\left[\partial_{\varphi} B_{0} \star_{\mathbf{c}} \varphi\right](\mathbf{l})\left[\partial_{\varphi} B_{0} \star_{\mathbf{c}} \varphi\right]\left(\mathbf{l}^{\prime}\right)\right\rangle \\
& +\left\langle\left[\partial_{\varphi} E_{0} \star_{\mathbf{S}} \varphi\right](\mathbf{l})\left[\partial_{\varphi} E_{0} \star_{\mathbf{S}} \varphi\right]\left(\mathbf{l}^{\prime}\right)\right\rangle
\end{aligned}
$$

to $O\left(\varphi^{2}\right)$.

Evaluating the convolutions and Wick expanding these correlators lead to the expressions

$$
\begin{aligned}
& \left\langle B_{0}(\mathbf{l})\left[\partial_{\varphi}^{2} B_{0} \star_{\mathbf{c}} \varphi \star \varphi\right]\left(\mathbf{I}^{\prime}\right)\right\rangle \\
& =\left\langle\left[\partial_{\varphi}^{2} B_{0} \star_{\mathbf{c}} \varphi \star \varphi\right]\left(\mathbf{I}^{\prime}\right) B_{0}(\mathbf{I})\right\rangle \\
& =(2 \pi)^{2} \delta^{2}\left(\mathbf{l}+\mathbf{l}^{\prime}\right)\left[\sigma^{(\varphi \varphi)} C_{l}^{B_{0} \partial^{2} B_{0}}\right], \\
& \left\langle\left[\partial_{\varphi} B_{0} \star_{\mathbf{c}} \varphi\right](\mathbf{I})\left[\partial_{\varphi} B_{0} \star_{\mathbf{c}} \varphi\right]\left(\mathbf{I}^{\prime}\right)\right\rangle \\
& =(2 \pi)^{2} \delta^{2}\left(\mathbf{I}+\mathbf{l}^{\prime}\right) \int \frac{d^{2} \mathbf{l}_{1}}{(2 \pi)^{2}} \cos ^{2}\left(2 \phi_{l_{1}}\right) \\
& \times C_{l_{1}}^{\partial B_{0} \partial B_{0}} C_{\left|\mathbf{1}-\mathbf{I}_{1}\right|}^{\varphi \varphi}, \\
& \left\langle\left[\partial_{\varphi} E_{0} \star_{\mathrm{S}} \varphi\right](\mathbf{l})\left[\partial_{\varphi} E_{0} \star_{\mathrm{S}} \varphi\right]\left(\mathbf{I}^{\prime}\right)\right\rangle \\
& =(2 \pi)^{2} \delta^{2}\left(\mathbf{I}+\mathbf{l}^{\prime}\right) \int \frac{d^{2} \mathbf{l}_{1}}{(2 \pi)^{2}} \\
& \times\left\{\sin ^{2}\left(2 \phi_{l_{1}}\right) C_{l_{1}}^{\partial E_{0} \partial E_{0}} C_{\left|\mathbf{I}-\mathbf{I}_{1}\right|}^{\varphi \varphi}+\sin \left(2 \phi_{l_{1}}\right)\right. \\
& \left.\times\left[\frac{l_{1}^{2} \sin \left(2 \phi_{l_{1}}\right)-2 l l_{1} \sin \left(\phi_{l_{1}}\right)}{\left|\mathbf{I}-\mathbf{l}_{1}\right|^{2}}\right] C_{l_{1}}^{\partial E_{0} \varphi} C_{\left|\mathbf{I}-\mathbf{l}_{1}\right|}^{\partial E_{0} \varphi}\right\} .
\end{aligned}
$$

Any $\varphi-B_{0}$ cross-correlations must vanish due to parity.

Collecting all terms we find that the expression for the average $B$-mode power spectrum is

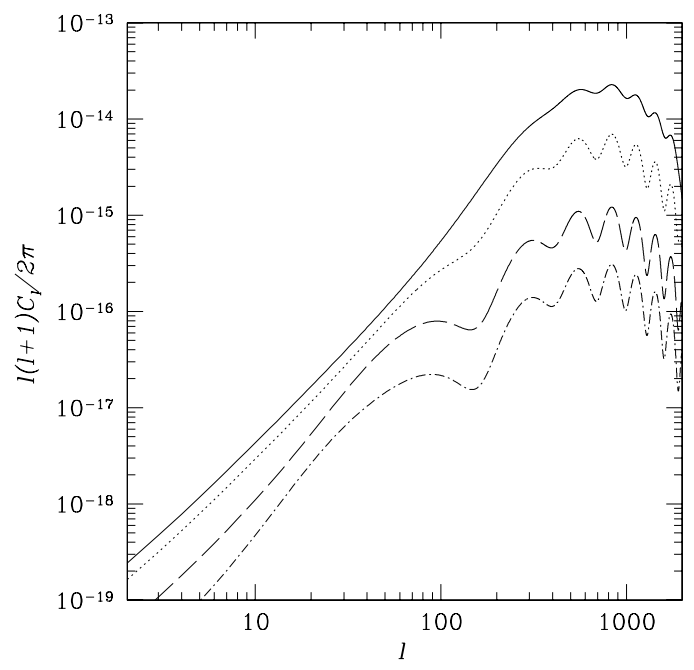

FIG. 9. The $B B$ power spectra for the Gaussian correlation function for $\varphi$ with $\sigma^{(\varphi \varphi)}=9 \times 10^{-4}$ and $\theta_{c}=1^{\circ}$ (solid line), $\theta_{c}=2^{\circ}$ (dotted line), $\theta_{c}=5^{\circ}$ (dashed line), and $\theta_{c}=10^{\circ}$ (dot-dashed line).

$$
\begin{aligned}
C_{l}^{B B}= & C_{l}^{B_{0} B_{0}}+\sigma^{(\varphi \varphi)} C_{l}^{B_{0} \partial^{2} B_{0}} \\
& +\int \frac{d^{2} \mathbf{l}^{\prime}}{(2 \pi)^{2}}\left\{\left[\cos ^{2}\left(2 \phi_{l^{\prime}}\right) C_{l^{\prime}}^{\partial B_{0} \partial B_{0}}\right.\right. \\
& \left.+\sin ^{2}\left(2 \phi_{l^{\prime}}\right) C_{l^{\prime}}^{\partial E_{0} \partial E_{0}}\right] C_{\left|\mathbf{l}-\mathbf{l}^{\prime}\right|}^{\varphi \varphi}+\sin \left(2 \phi_{l^{\prime}}\right) \\
& \left.\times\left[\frac{l^{\prime 2} \sin \left(2 \phi_{l^{\prime}}\right)-2 l l^{\prime} \sin \left(\phi_{l^{\prime}}\right)}{\left|\mathbf{l}-\mathbf{l}^{\prime}\right|^{2}}\right] C_{l^{\prime}}^{\partial B_{0} \varphi} C_{\left|\mathbf{I}-\mathbf{l}^{\prime}\right|}^{\partial B_{0} \varphi}\right\},
\end{aligned}
$$

and if $E_{0}$ and $\varphi$ are uncorrelated the expression simplifies to

$$
\begin{aligned}
C_{l}^{B B}= & C_{l}^{B_{0} B_{0}}+\sigma^{(\varphi \varphi)} C_{l}^{B_{0} \partial^{2} B_{0}}+\int \frac{d^{2} \mathbf{I}^{\prime}}{(2 \pi)^{2}}\left[\cos ^{2}\left(2 \phi_{l^{\prime}}\right) C_{l^{\prime}}^{\partial B_{0} \partial B_{0}}\right. \\
& \left.+\sin ^{2}\left(2 \phi_{l^{\prime}}\right) C_{l^{\prime}}^{\partial E_{0} \partial E_{0}}\right] C_{\left|\mathbf{I}^{-} \mathbf{I}^{\prime}\right|}^{\varphi \varphi} .
\end{aligned}
$$

If the intrinsic $B$ modes due to gravitational waves are negligible then for our $\varphi$ power spectrum, Eq. (64), the average $B$-mode power spectrum is

$$
\begin{aligned}
C_{l}^{B B}= & \sigma^{(\varphi \varphi)}\left\{\frac { \theta _ { c } ^ { 2 } } { 4 } \int d l ^ { \prime } l ^ { \prime } e ^ { - ( 1 / 4 ) ( l ^ { 2 } + l ^ { \prime 2 } ) \theta _ { c } ^ { 2 } } \left[I_{0}\left(\frac{\theta_{c}^{2}}{2} l l^{\prime}\right)\right.\right. \\
& \left.\left.-I_{4}\left(\frac{\theta_{c}^{2}}{2} l l^{\prime}\right)\right] C_{l^{\prime}}^{\partial E_{0} \partial E_{0}}\right\} .
\end{aligned}
$$

This expression shows that $E$ modes modulated by $\varphi$ fluctuations can induce $B$ modes. We note here that this effect is more general than the specific application of the $\alpha$ variation that we focus on here, and is a generic feature of any fluctuations that modulate the power spectrum but have negligible effects on the evolution of the dominant density perturbations. In Fig. 9 we plot the induced $B$-mode power spectra due to $\varphi$ fluctuations. The induced $B$-mode power spectrum 
inherits the oscillatory features of the unperturbed $E_{0}$-mode power spectrum as long as the correlation angle is larger than the horizon size at recombination, while the amplitude of the power spectrum decreases as the correlation angle increases.

\section{BISPECTRA}

We have shown in the previous section that modulation of the temperature and polarization power spectra by spatial variations of $\alpha$ alters the mean power spectra of CMB anisotropies. In this section and the one that follows we show how this modulation introduces higher order correlations into the CMB temperature field.

The temperature bispectrum is defined in terms of the connected piece (the terms remaining after the Gaussian piece is subtracted out) of the three-point correlation function in Fourier space as

$$
\left\langle\Theta\left(\mathbf{l}_{1}\right) \Theta\left(\mathbf{l}_{2}\right) \Theta\left(\mathbf{l}_{3}\right)\right\rangle_{c}=(2 \pi)^{2} \delta^{2}\left(\mathbf{l}_{1}+\mathbf{l}_{2}+\mathbf{l}_{3}\right) B^{\Theta \Theta \Theta}\left(\mathbf{l}_{1}, \mathbf{l}_{2}, \mathbf{l}_{3}\right) .
$$

This expression must be invariant under the exchange of any two fields, or equivalently, l vectors. We insert Eq. (49) into the three-point correlation function, and after some straightforward algebra we find that

$$
B^{\Theta \Theta \Theta}\left(\mathbf{l}_{1}, \mathbf{l}_{2}, \mathbf{l}_{3}\right)=\sum_{\substack{i, j=1 \\ i<j}}^{3} \mathrm{~B}\left(\mathbf{l}_{i}, \mathbf{l}_{j}, \mathbf{l}_{6-i-j}\right),
$$

where the sums runs over the distinct permutations of $\{1,2,3\}$ and, to leading order,

$$
\mathrm{B}\left(\mathbf{l}_{i}, \mathbf{l}_{j}, \mathbf{l}_{k}\right)=C_{l_{i}}^{\Theta_{0} \partial \Theta_{0}} C_{l_{j}}^{\Theta_{0} \varphi}+C_{l_{i}}^{\Theta_{0} \varphi} C_{l_{j}}^{\Theta_{0} \partial \Theta_{0}} .
$$

Thus the temperature bispectrum vanishes unless $\varphi$ and $\Theta_{0}$ are correlated. In the models we consider in this work, $\varphi$ and $\Theta_{0}$ are not expected to be highly correlated. However, if a model of spatial variations of $\alpha$ predicts that variations of $\varphi$ and $\Theta_{0}$ are strongly correlated the bispectrum may be a strong signature of such a model as, unlike the power spectrum or the trispectrum (discussed below), it is first order in $\varphi$. We note that expressions analogous to Eqs. (88) and (89) will hold for the polarization and cross-bispectra as well.

\section{TRISPECTRA}

In analogy with the bispectrum, the temperature trispectrum is defined in terms of the connected piece of the fourpoint correlation function in Fourier space as

$$
\begin{aligned}
& \left\langle\Theta\left(\mathbf{l}_{1}\right) \Theta\left(\mathbf{l}_{2}\right) \Theta\left(\mathbf{l}_{3}\right) \Theta\left(\mathbf{l}_{4}\right)\right\rangle_{c} \\
& =(2 \pi)^{2} \delta^{2}\left(\mathbf{l}_{1}+\mathbf{l}_{2}+\mathbf{l}_{3}+\mathbf{l}_{4}\right) T^{\Theta \Theta \Theta \Theta}\left(\mathbf{l}_{1}, \mathbf{l}_{2}, \mathbf{l}_{3}, \mathbf{l}_{4}\right) .
\end{aligned}
$$

This expression must also be invariant under the exchange of any two fields, or equivalently, l vectors. We insert Eq. (49) into the four-point correlation function, and after some straightforward algebra we find that

$$
\begin{aligned}
T^{\Theta \Theta \Theta \Theta}\left(\mathbf{l}_{1}, \mathbf{l}_{2}, \mathbf{l}_{3}, \mathbf{l}_{4}\right)= & \sum_{\substack{i, j=1 \\
i<j}}^{4} \sum_{\substack{k, l=1(\neq i, j) \\
k<l}}^{4} \mathrm{~T}_{\mathrm{A}}\left(\mathbf{l}_{i}, \mathbf{l}_{j}, \mathbf{l}_{k}, \mathbf{l}_{l}\right) \\
& +\sum_{\substack{i, j, k=1 \\
i<j<k}}^{4} \sum_{\substack{l=1 \\
l \neq i, j, k}}^{4} \mathrm{~T}_{\mathrm{B}}\left(\mathbf{l}_{i}, \mathbf{l}_{j}, \mathbf{l}_{k}, \mathbf{l}_{l}\right),
\end{aligned}
$$

where the sums run over the distinct permutations of $\{1,2,3,4\}$,

$$
\begin{aligned}
\mathrm{T}_{\mathrm{A}}\left(\mathbf{l}_{i}, \mathbf{l}_{j}, \mathbf{l}_{k}, \mathbf{l}_{l}\right)= & C_{l_{i}}^{\Theta_{0} \partial \Theta_{0}} C_{l_{j}}^{\Theta_{0} \partial \Theta_{0}}\left(C_{\left|\mathbf{l}_{j}+\mathbf{l}_{k}\right|}^{\varphi \varphi}+C_{\left|\mathbf{l}_{j}+\mathbf{l}_{l}\right|}^{\varphi \varphi}\right) \\
& +\left(C_{l_{i}}^{\Theta_{0} \partial \Theta_{0}} C_{l_{j}}^{\Theta_{0} \varphi}+C_{l_{i}}^{\Theta_{0} \varphi} C_{l_{j}}^{\Theta_{0} \partial \Theta_{0}}\right)\left(C_{\left|\mathbf{l}_{j}+\mathbf{l}_{k}\right|}^{\partial \Theta_{0} \varphi}\right. \\
& \left.+C_{\left|\mathbf{l}_{j}+\mathbf{l}_{l}\right|}^{\partial \Theta_{0} \varphi}\right)+C_{l_{i}}^{\Theta_{0} \varphi} C_{l_{j}}^{\Theta_{0} \varphi}\left(C_{\left|\mathbf{l}_{j}+\mathbf{l}_{k}\right|}^{\partial \Theta_{0} \partial \Theta_{0}}+C_{\left|\mathbf{l}_{j}+\mathbf{l}_{l}\right|}^{\partial \Theta_{0} \partial \Theta_{0}}\right)
\end{aligned}
$$

and

$$
\begin{aligned}
\mathrm{T}_{\mathrm{B}}\left(\mathbf{l}_{i}, \mathbf{l}_{j}, \mathbf{l}_{k}, \mathbf{l}_{l}\right)= & 2 C_{l_{i}}^{\Theta_{0} \partial^{2} \Theta_{0}} C_{l_{j}}^{\Theta_{0} \varphi} C_{l_{k}}^{\Theta_{0} \varphi} \\
& +2 C_{l_{i}}^{\Theta_{0} \varphi}\left(C_{l_{j}}^{\Theta_{0} \varphi} C_{l_{k}}^{\Theta_{0} \partial^{2} \Theta_{0}}+C_{l_{j}}^{\Theta_{0} \partial^{2} \Theta_{0}} C_{l_{k}}^{\Theta_{0} \varphi}\right)
\end{aligned}
$$

If $\Theta_{0}$ and $\varphi$ have no cross-correlation, the second term vanishes and the first term simplifies to

$$
\mathrm{T}_{\mathrm{A}}\left(\mathbf{l}_{i}, \mathbf{l}_{j}, \mathbf{l}_{k}, \mathbf{l}_{l}\right)=C_{l_{i}}^{\Theta_{0} \partial \Theta_{0}} C_{l_{j}}^{\Theta_{0} \partial \Theta_{0}}\left(C_{\left|\mathbf{l}_{j}+\mathbf{l}_{k}\right|}^{\varphi \varphi}+C_{\left|\mathbf{l}_{j}+\mathbf{l}_{l}\right|}^{\varphi \varphi}\right) .
$$

Expressions similar to Eqs. (91)-(93) will hold for the polarization and cross-trispectra as well.

\section{A. The kurtosis}

The trispectrum is a nontrivial function of six variables and extracting the full trispectrum from CMB data will be a challenging experimental endeavor. It is therefore worthwhile to examine simpler statistical signatures of nonGaussianity in the CMB. If the trispectrum is nonvanishing the probability distribution function of $\Theta(\hat{\mathbf{n}})$ on the sky will no longer be precisely Gaussian. As in the case of weak lensing, the deviation from Gaussianity can be parametrized by the kurtosis [48], which is a measure of how flattened out or peaked the distribution is relative to a Gaussian distribution.

The kurtosis of a non-Gaussian random field may be written in terms of the trispectrum as

$$
\begin{aligned}
K(\theta)= & \frac{1}{\sigma^{4}(\theta)} \int \frac{d^{2} \mathbf{l}_{1}}{(2 \pi)^{2}} \frac{d^{2} \mathbf{l}_{2}}{(2 \pi)^{2}} \frac{d^{2} \mathbf{l}_{3}}{(2 \pi)^{2}} \frac{d^{2} \mathbf{l}_{4}}{(2 \pi)^{2}} \\
& \times\left\{(2 \pi)^{2} \delta^{2}\left(\mathbf{l}_{1}+\mathbf{l}_{2}+\mathbf{l}_{3}+\mathbf{l}_{4}\right) T^{\Theta \Theta \Theta \Theta}\right. \\
& \left.\times\left(\mathbf{l}_{1}, \mathbf{l}_{2}, \mathbf{l}_{3}, \mathbf{l}_{4}\right) W\left(l_{1} \theta\right) W\left(l_{2} \theta\right) W\left(l_{3} \theta\right) W\left(l_{4} \theta\right)\right\},
\end{aligned}
$$




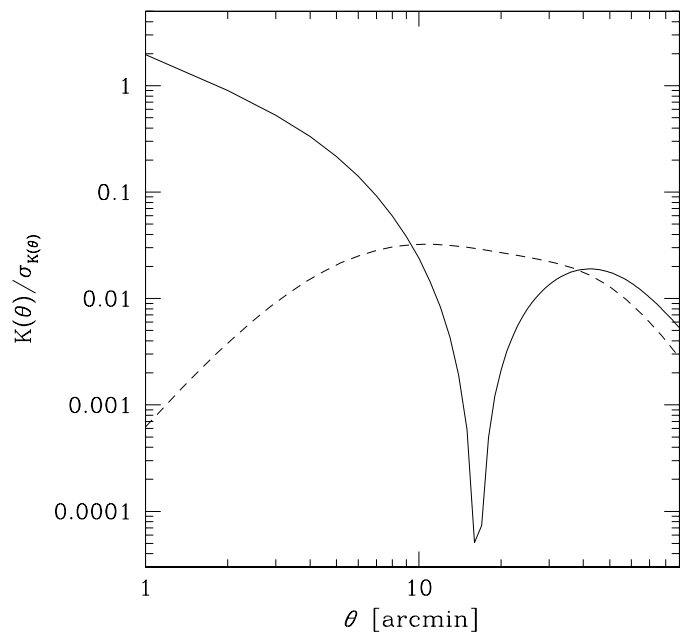

FIG. 10. The ratio of the kurtosis to the variance in the estimator of the kurtosis due to Gaussian fluctuations for spatial $\alpha$ fluctuations with $\sigma^{(\varphi \varphi)}=9 \times 10^{-4}$ and $\theta_{c}=1^{\circ}$ (solid line) and the same ratio for weak lensing (dashed line). The kurtosis is undetectable for weak lensing at any angular resolution, while for spatial $\alpha$ fluctuations of this amplitude the kurtosis may in principle be detected by a high resolution no-noise experiment.

where $W(l \theta)$ is a smoothing function with smoothing scale $\theta$, and

$$
\sigma^{2}(\theta)=\int \frac{d^{2} \mathbf{l}}{(2 \pi)^{2}} C_{l}^{\Theta \Theta} W^{2}(l \theta)
$$

is the smoothed variance.

If we adopt a Gaussian smoothing function

$$
W(l \theta)=e^{-(1 / 2) \sigma_{b}^{2} l^{2}}
$$

with $\sigma_{b}=\theta /(\sqrt{8 \ln 2})$ and insert the expression for the trispectrum from Eq. (94) we find after some algebra that

$$
\begin{aligned}
K(\theta)= & \frac{3}{2 \pi^{3} \sigma^{4}(\theta)} \int l_{1} d l_{1} l_{2} d l_{2} l_{3} d l_{3}\left\{C_{l_{1}}^{\Theta \partial \Theta} C_{l_{2}}^{\varphi \varphi} C_{l_{3}}^{\Theta \partial \Theta}\right. \\
& \left.\times\left[I_{0}\left(\sigma_{b}^{2} l_{1} l_{2}\right) I_{0}\left(\sigma_{b}^{2} l_{2} l_{3}\right) e^{-\sigma_{b}^{2}\left(l_{1}^{2}+l_{2}^{2}+l_{3}^{2}\right)}\right]\right\} .
\end{aligned}
$$

It was shown in Ref. [48] that the sample variance of the kurtosis of a Gaussian random field smoothed over an angular scale $\theta$ for a full-sky experiment is

$$
\sigma_{K(\theta)}^{2}=\frac{3}{2} \theta^{2} .
$$

In Fig. 10 we plot the ratio of the kurtosis to the standard deviation of the kurtosis as a function of smoothing scale. Despite the improved variance at high resolution the kurtosis due to weak lensing cannot be detected because in the limit of infinite resolution the kurtosis of weak lensing vanishes more quickly than the variance. This can be understood by noting that weak lensing is power conserving because it maps the temperature at one point on the sky to another, and so with infinite resolution the probability distribution func- tion is Gaussian. In contrast, the kurtosis due to spatial $\alpha$ fluctuations approaches a constant value in the limit of infinite resolution, and so for low $\theta$ the signal-to-noise increases like $\theta^{-1}$. This occurs because $\alpha$ fluctuations do not lead to a pure remapping of the temperature pattern (they do not conserve power), and thus in the limit of infinite resolution modulate the variance of the (Gaussian) temperature probability distribution function from one patch of the sky to another. The resulting mean probability distribution is no longer Gaussian. Note that the dip in the kurtosis at $\theta$ $\approx 15$ arcmin occurs because when smoothed over that scale modulation of the CMB due to spatial $\alpha$ fluctuations becomes approximately power conserving. An ideal noise-free experiment at an angular resolution of 1 arcmin could detect the kurtosis due to $\alpha$ fluctuations at the level of $\sigma^{(\varphi \varphi)}=9$ $\times 10^{-4}$, while higher resolution experiments could observe lower amplitude $\alpha$ fluctuations.

\section{B. A discriminating filter}

As discussed above, weak lensing of the CMB by matter along the line of sight must induce a contribution to the trispectrum

$$
L^{\Theta \Theta \Theta \Theta}\left(\mathbf{l}_{1}, \mathbf{l}_{2}, \mathbf{l}_{3}, \mathbf{l}_{4}\right)=\sum_{\substack{i, j=1 \\ i<j}}^{4} \sum_{\substack{k, l=1(\neq i, j) \\ k<l}}^{4} \mathrm{~L}\left(\mathbf{l}_{i}, \mathbf{l}_{j}, \mathbf{l}_{k}, \mathbf{l}_{l}\right),
$$

where

$$
\begin{aligned}
\mathrm{L}\left(\mathbf{l}_{i}, \mathbf{l}_{j}, \mathbf{l}_{k}, \mathbf{l}_{l}\right)= & -C_{l_{k}}^{\Theta_{0} \Theta_{0}} C_{l_{l}}^{\Theta_{0} \Theta_{0}}\left\{C_{\left|\mathbf{l}_{i}+\mathbf{l}_{k}\right|}^{\phi \phi}\left[\left(\mathbf{l}_{i}+\mathbf{l}_{k}\right) \cdot \mathbf{l}_{k}\right]\right. \\
& \times\left[\left(\mathbf{l}_{i}+\mathbf{l}_{k}\right) \cdot \mathbf{l}_{l}\right]+C_{\left|\mathbf{l}_{j}+\mathbf{l}_{k}\right|}^{\phi \phi}\left[\left(\mathbf{l}_{j}+\mathbf{l}_{k}\right) \cdot \mathbf{l}_{k}\right] \\
& \times\left[\left(\mathbf{l}_{j}+\mathbf{l}_{k}\right) \cdot \mathbf{l}_{l}\right\} .
\end{aligned}
$$

We emphasize that in this expression $C_{l}^{\phi \phi}$ is the power spectrum of the projected lensing potential and not the power spectrum of $\alpha$ fluctuations $C_{l}^{\varphi \varphi}$.

Since this contribution to the trispectrum must be in the $\mathrm{CMB}$, we now derive the a filter to distinguish between the weak-lensing and the $\alpha$-fluctuation trispectra.

For compactness we introduce the notation

$$
X_{i j k} \equiv X^{\Theta \Theta \Theta \Theta}\left(\mathbf{l}_{i}, \mathbf{l}_{j}, \mathbf{l}_{k},-\mathbf{l}_{i}-\mathbf{l}_{j}-\mathbf{l}_{k}\right),
$$

and

$$
\sum_{i j k} \equiv \int \frac{d^{2} \mathbf{l}_{i}}{(2 \pi)^{2}} \frac{d^{2} \mathbf{l}_{j}}{(2 \pi)^{2}} \frac{d^{2} \mathbf{l}_{k}}{(2 \pi)^{2}}
$$

The signal for detection of the $\alpha$-fluctuation trispectrum can be in general written as a windowed integral over trispectrum configurations (quadrilaterals in I-space),

$$
S=\sum_{i j k} W_{i j k} T_{i j k}
$$


where $W_{i j k}$ is the to-be-determined window function.

To determine the least-square quartic discriminator we treat the trispectrum due to weak gravitational lensing as a source of noise that should be minimized and write

$$
N_{L}=\sum_{i j k} W_{i j k} L_{i j k}
$$

In addition we at minimum also have the Gaussian noise due to cosmic variance

$$
N_{G}^{2}=\sum_{i j k} W_{i j k}^{2} G_{i j k},
$$

where

$$
G_{i j k}=\frac{4 !}{4 \pi f_{s k y}} C_{l_{i}}^{\widehat{\Theta \Theta}} C_{l_{j}}^{\widehat{\Theta \Theta}} C_{l_{k}}^{\widehat{\Theta \Theta}} C_{\left|\mathbf{l}_{i}+\mathbf{l}_{j}+\mathbf{l}_{k}\right|}^{\widehat{\Theta \Theta}},
$$

and

$$
C_{l}^{\widehat{\Theta \Theta}}=C_{l}^{\Theta \Theta}+\frac{4 \pi f_{s k y} s^{2}}{t_{\text {exp }} T_{C M B}^{2}} e^{\sigma_{b}^{2} l^{2}}
$$

is the sum of the actual CMB power spectrum and the noise power spectrum introduced by an experiment that observes a fraction $f_{s k y}$ of the sky with beam width $\sigma_{b}$ for a time $t_{\text {exp }}$ with detectors of noise-equivalent-temperature $s$.

The total noise is then just

$$
N^{2}=N_{G}^{2}+N_{L}^{2}
$$

We want to solve for the $W_{i j k}$ that maximizes the signal to noise ratio $S / N$. To do this we set

$$
\frac{\delta}{\delta W_{i j k}}\left(\frac{S^{2}}{N^{2}}\right)=0
$$

After some straightforward algebra, and making use of the fact that $S^{2} / N^{2}$ is invariant under renormalization of $W_{i j k}$, we find that the signal-to-noise ratio is extremized only if $W_{i j k}$ takes the form

$$
W_{i j k}=\frac{T_{i j k}-\lambda L_{i j k}}{G_{i j k}} .
$$

Thus we have

$$
\frac{S^{2}}{N^{2}}=\frac{\mathcal{T}^{2}-2 \lambda \mathcal{T} \mathcal{X}+\lambda^{2} \mathcal{X}^{2}}{\mathcal{T}+\mathcal{X}^{2}-\left(2 \lambda \mathcal{X}-\lambda^{2} \mathcal{L}\right)(1+\mathcal{L})},
$$

where

$$
\begin{aligned}
& \mathcal{T}=\sum_{i j k} \frac{T_{i j k}^{2}}{G_{i j k}}, \\
& \mathcal{X}=\sum_{i j k} \frac{L_{i j k} T_{i j k}}{G_{i j k}},
\end{aligned}
$$

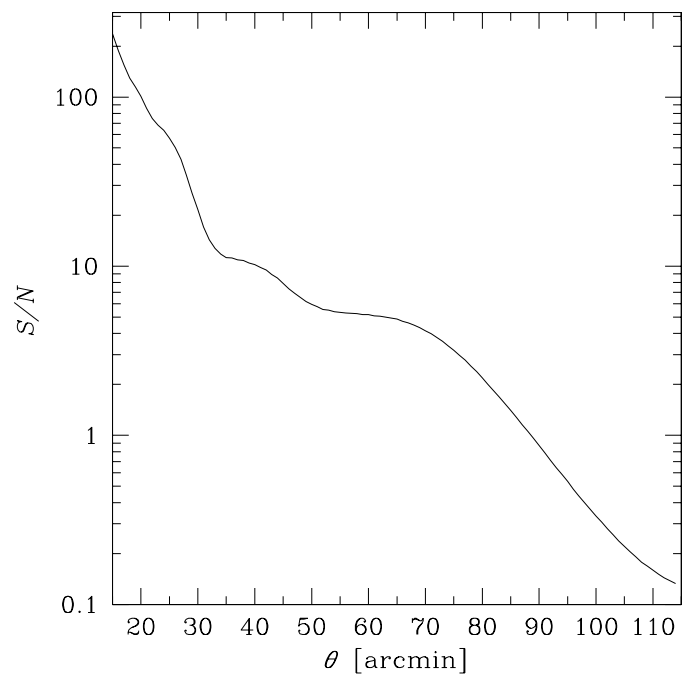

FIG. 11. The signal-to-noise ratio of the $\alpha$-fluctuation trispectrum that would be observed by an experiment with $s$ $=12.42 \mu \mathrm{K} \sqrt{\mathrm{sec}}$ observing for 1 year (the effective parameters of the Planck satellite) with resolution $\theta$ if weak lensing were not present for $\sigma_{(\varphi \varphi)}=9 \times 10^{-4}$. Since in practice we know weak lensing must be present in the CMB this curve serves as an upper bound on the observable signal-to-noise ratio.

$$
\mathcal{L}=\sum_{i j k} \frac{L_{i j k}^{2}}{G_{i j k}}
$$

and the optimum weighting is

$$
\lambda=\frac{\mathcal{X}}{1+\mathcal{L}}
$$

In the absence of lensing this expression reduces to the conventional signal-to-noise measure

$$
\frac{S^{2}}{N^{2}}=\mathcal{T}=\sum_{i j k} \frac{T_{i j k}^{2}}{G_{i j k}} .
$$

In Fig. 11 we show the maximum signal-to-noise ratio for detection of the $\alpha$-fluctuation trispectrum that would be detected were weak lensing not present. For a given weak lensing power spectrum $C_{l}^{\phi \phi}$ the actual signal to noise will be less than the bound shown.

\section{THEORETICAL MODELS OF VARIABLE $\boldsymbol{\alpha}$}

Recent theoretical work [11-16,18-21] has considered the ingredients required of field-theory models for a variable fine-structure parameter in order to explain a small time variation of $\alpha$. Here we briefly amend those discussions to consider the field-theory requirements for spatial variations of the sort we consider in this paper.

The simplest way to introduce spatial variation of $\alpha$ is to couple the photon to a scalar field $\phi(x)$ through a term (see, e.g., Ref. [20] and references therein) 


$$
\mathcal{L}_{\phi \gamma \gamma}=-\frac{\lambda}{4} g\left(\frac{\phi}{f_{\phi}}\right) F^{\mu \nu} F_{\mu \nu},
$$

in the Lagrangian that appears in addition to the usual electromagnetic Lagrangian, $F^{\mu \nu} F_{\mu \nu} / 4$. Here, $f_{\phi}$ is a constant with dimensions of mass, $\lambda$ is an overall coupling constant, and $g(x)=\sum_{n=1}^{\infty} c_{n} x^{n}$ is a dimensionless function of the ratio $\phi / f_{\phi}$ normalized so that $g(1)=1$. This interaction then leads to a fractional change in $\alpha$,

$$
\frac{\delta \alpha}{\alpha}=-\lambda g\left(\frac{\phi}{f_{\phi}}\right)=\varphi \text {. }
$$

The best present constraint to $g$ comes from the evolution of globular-cluster stars; this requires $[\lambda(d g / d \phi)]^{-1} \gtrsim 1.6$ $\times 10^{10} \mathrm{GeV}$ [49]. If we consider fractional fluctuations in $\alpha$ of $\lesssim 10^{-2}$, then $\lambda \sim 10^{-2}, \delta \phi \lesssim f_{\phi}$, and $\sigma^{(\varphi \varphi)} \sim \lambda^{2}$.

Cosmological mechanisms that might induce spatial variations in $\phi$ are analogous to those that have been considered, e.g., for spatial variations in the axion field [50]. These variations in $\phi$ can arise either during or after inflation. If $\phi$ is a spectator field during inflation, then fluctuations in $\phi$ can be induced quantum mechanically during inflation resulting in a nearly scale-invariant spectrum for $\phi$ fluctuations. Alternatively, if $\phi$ is a pseudo-Nambu-Goldstone field in a model with an approximate global symmetry, it could fall during the symmetry-breaking phase transition to different random points on the vacuum manifold (which is periodic in $\phi$ with period $2 f_{\phi}$ ) in different causally disconnected regions. In this case, the scalar-photon interaction will most generally be an arbitrary Fourier series in $\phi$. A simple interaction of this form, containing only the first harmonic and (as motivated below) constraining $g\left(\phi / f_{\phi}\right)$ to be an even function, is

$$
\mathcal{L}_{\phi \gamma \gamma}=-\frac{\lambda}{8}\left[1-\cos \left(\frac{\pi \phi}{f_{\phi}}\right)\right] F^{\mu \nu} F_{\mu \nu} .
$$

For such an interaction $\phi$ will be uncorrelated on scales larger than the horizon, and $\delta \alpha / \alpha$ is fixed by the magnitude of the explicit symmetry breaking term $\lambda$, rather than by $f_{\phi}$. In either case (inflation or spontaneous symmetry breaking), the gradient term in the scalar-field Lagrangian will tend to align the scalar field within causally connected regions of the Universe. Thus, the value of $\alpha$ at the surface of last scatter should be constant within square-degree patches, but will vary from one square-degree patch to another, as we have assumed throughout this paper.

Now consider constraints to the potential-energy density $V(\phi)$ for the scalar field. If it is quadratic, $V(\phi)$ $=m_{\phi}^{2} \phi^{2} / 2$, then (neglecting for the moment the interaction with photons) the equation of motion for $\phi$ is

$$
\ddot{\phi}+3 H \dot{\phi}+m_{\phi}^{2} \phi=0
$$

and has the solution

$$
\phi(t)=\phi_{0} \frac{\sin \left(m_{\phi} t\right)}{m_{\phi} t}
$$

in the matter dominated era. At early times when $H \gg m_{\phi}$ the value of $\phi$ is frozen at $\phi \approx \phi_{0}$, while for $H \lesssim m_{\phi}$ the field in each horizon will oscillate about its minimum with an amplitude decaying like $1 / t$. We thus require $m_{\phi} \lesssim H_{\text {rec }}$ $\simeq 10^{-28} \mathrm{eV}$ so that the $\alpha$ fluctuations are frozen in at recombination. On the other hand, observations of the Lyman- $\alpha$ forest at redshifts $z \sim 4$ show no evidence of spatial fluctuations in $\alpha$ larger than one part in $10^{4}$. Thus, if we consider $(\delta \alpha / \alpha) \geq 10^{-4}$ at the surface of last scatter, then we must require that $\left.\lambda g\right|_{z \approx 4} \lesssim 10^{-4}$. If the leading order term in $g$ is quadratic in $\phi$ (as we argue below) this leads to the constraint $m_{\phi} \gtrsim \sqrt{\lambda / 0.01} \times 10^{-31} \mathrm{eV}$. Finally, laboratory experiments constrain the value $\dot{\alpha} / \alpha$ today to be $\lesssim 5$ $\times 10^{-15} \mathrm{yr}^{-1}$ [51]. To satisfy this requirement we require that $g \propto \phi^{2}$ to leading order at small $\phi$ so that $\dot{\alpha} / \alpha \propto t^{-2}$. This leads to the constraint $m_{\phi} \gtrsim 2 \times(\lambda / 0.01) \times 10^{-30} \mathrm{eV}$ if the oscillatory factor is unity. We note that if $g \propto \phi^{n}$ with $n>2$ for small $\phi$ this constraint can be relaxed, and that late-time $\Lambda$ domination does not significantly alter the limit. These constraints will ensure that the model conforms to upper limits to $\alpha$ variations in the low-redshift Universe, but we caution that they are constraints based on the root-mean-squared value of the scalar field. If we happen to live in a region where the amplitude of $\phi$ is randomly small (large) the constraints based on local observations will be weaker (stronger) than discussed above. Also, the nonlinear evolution of the mass distribution may modify constraints based on recent terrestrial phenomena [52].

We must also be sure that fluctuations in the scalar field do not lead to density perturbations that exceed those of amplitude $10^{-5}$. This constraint requires that the gradientenergy density, $k^{2}(\Delta \phi)^{2} \lesssim 10^{-5} \rho_{m}\left(z_{\text {rec }}\right)$, where $k \simeq H_{\text {rec }}$ is the largest wave number for which perturbations are significant at the redshift $z_{\mathrm{rec}} \simeq 1100$ of decoupling, and $H_{\mathrm{rec}}$ $=\Omega_{m}^{1 / 2} H_{0}\left(1+z_{\text {rec }}\right)^{3 / 2}$ is the Hubble parameter at decoupling. However, from the Friedmann equation, $\rho_{m}\left(z_{\mathrm{rec}}\right) \sim H_{\mathrm{rec}}^{2} m_{\mathrm{Pl}}^{2}$, where $m_{\mathrm{Pl}} \simeq 10^{19} \mathrm{GeV}$ is the Planck mass, so we find a constraint $\Delta \phi \lesssim f_{\phi} \lesssim 3 \times 10^{16} \mathrm{GeV}$.

Now lets examine the effect of the scalar-photon Lagrangian on the scalar field dynamics. If we include the interaction in the Lagrangian it is easy to verify the equation of motion for the scalar field is modified to include a forcing term

$$
\ddot{\phi}+3 H \dot{\phi}+m_{\phi}^{2} \phi+\frac{\lambda}{4} \frac{d g}{d \phi}\left\langle F_{\mu \nu} F^{\mu \nu}\right\rangle=0,
$$

where

$$
\left\langle F_{\mu \nu} F^{\mu \nu}\right\rangle=\frac{1}{8 \pi}\left(\left\langle E^{2}\right\rangle-\left\langle B^{2}\right\rangle\right)
$$

and $\left\langle E^{2}\right\rangle$ and $\left\langle B^{2}\right\rangle$ are the spatially averaged squared electric and magnetic fields, respectively. A bath of thermal photons has $\left\langle E^{2}\right\rangle=\left\langle B^{2}\right\rangle$ and so will not contribute to the forcing term. However, nonrelativistic matter is a source for electromagnetic fields with $\left\langle E^{2}\right\rangle \neq\left\langle B^{2}\right\rangle$, and so the forcing term should be proportional to the density of nonrelativistic matter 
$\rho_{m}$. If, as we discussed above, $g\left(\phi / f_{\phi}\right)$ is dominated by its quadratic term then in the matter dominated era the equation of motion reads

$$
\ddot{\phi}+\frac{2}{t} \dot{\phi}+\left(m_{\phi}^{2}+\frac{\eta}{t^{2}}\right) \phi=0
$$

where $\eta=\left(2 \lambda \xi \rho_{m_{0}} t_{0}^{2}\right) / f_{\phi}^{2}$, and $\xi$ is the fraction of matter density $\rho_{m}$ due to electromagnetic energy. ${ }^{1}$ Thus, if $\eta$ is small the effect of the forcing term is to introduce a small time-dependent mass term into the equation of motion. The solution will qualitatively behave like that of Eq. (122), except that the time that field begins to oscillate may shift by a small $\eta$-dependent factor, and the power law of decay will shift from -1 to $-1+\eta$. Generically $\eta$ need not be small, but for $f_{\phi} \gtrsim 5 \times 10^{15} \mathrm{GeV}$ and $\lambda \approx 0.01$ we find $|\eta| \lesssim 0.1$. $^{2}$ This brings us uncomfortably close to the limits on $f_{\phi}$ from gradient energy density. In a forthcoming publication [53] we argue that, in general, $\eta$ should be replaced by the quantity $\tilde{\eta}=\eta+\omega$, and that $\tilde{\eta}$ may be small even if $\eta$ is not. The term $\omega$ arises from the terms of the effective nonrenormalizable Lagrangian of the form $\lambda_{n} m_{\psi} \phi^{2 n} \bar{\psi} \psi$ or $\sigma_{n} m_{\chi}^{2} \phi^{2 n} \chi^{2}$ that couple the scalar field $\phi$ to the energy density in other matter fields. We note in passing that for those theories that do not lead to "fifth forces" which violate the weak-equivalence-principle (WEP) $\tilde{\eta}=0$ precisely.

We thus have a constraint $[\lambda(d g / d \phi)]^{-1} \gtrsim 10^{10} \mathrm{GeV}$ to the scalar-photon coupling, and a constraint $(2 \lambda / 0.01)$ $\times 10^{-30} \mathrm{eV} \leqslant m_{\phi} \leq 10^{-28} \mathrm{eV}$ to the scalar-particle mass. We note that these relations may seem hard to reconcile, as contributions to the scalar-particle propagator from divergent loop diagrams containing photons should generically be large. Likewise, if $\phi$ is a Goldstone mode for some spontaneously broken global symmetry, it may be unusual from the

\footnotetext{
${ }^{1}$ Naively, one might guess that $\sim \mathrm{nG}$ intergalactic magnetic fields would suggest $\xi \sim 10^{-11}$. However, the quantity of interest here is $\left\langle B^{2}\right\rangle$, rather than $\langle B\rangle^{2}$, as well as $\left\langle E^{2}\right\rangle$. Thus, strong microscopic magnetic fields in the vicinity of electrons and protons may contribute $\xi_{b} \sim 10^{-5}[20]$.

${ }^{2}$ This assumes a baryon fraction $\Omega_{b} / \Omega_{m}=1 / 6$, that magnetic energy contributes $\left|\xi_{b}\right| \approx 2 \times 10^{-5}[20]$, and that the dark matter has negligible electromagnetic energy so that $\xi=\left(\Omega_{b} / \Omega_{m}\right) \xi_{b}$.
}

point of view of Planck-scale physics [54]. It was also pointed out in Ref. [55] that variations of a light scalar should give rise to large variations in vacuum energy density. On the other hand, similar problems arise in models for inflation and for scalar-field models for dark energy and dark matter. Here, we take the view that our $\phi$-photon coupling is simply a low-energy effective Lagrangian, and anticipate that the light mass can be protected, e.g., by a mechanism such as that proposed in Ref. [56] where the scale of an inflation potential is fixed by the size of an extra dimension. If so then one might assume that whatever mechanism alleviates the cosmological-constant problem may also alleviate problems associated with vacuum-energy gradients.

\section{CONCLUSION}

In this paper we have studied the effects on the CMB of spatial fluctuations in the fine-structure parameter between causally disconnected regions of the Universe at the time of recombination. Although we have focused on the particular case of fluctuations in the fine-structure parameter, the formalism we have presented may be applied mutatis mutandis to other modifications of recombination physics that do not alter the evolution of the dominant density perturbations. As discussed above, such fluctuations will alter the predicted CMB power spectra, introduce a $B$-mode polarization signal, and introduce temperature and polarization trispectra and perhaps bispectra. We stress here that these results are not dependent on the particular model for $\alpha$ variation discussed in Sec. VII.

From the point of view of effective field theory, variations of the fine-structure parameter can be phrased in terms of a scalar-photon interaction Lagrangian $\mathcal{L}_{\phi \gamma \gamma}$, the parameters of which can be chosen to be consistent with current experimental limits. Ultimately, if such light, cosmologically interesting, scalars do exist in nature their mass must be protected by some yet unknown mechanism.

\section{ACKNOWLEDGMENTS}

We thank A. Cooray and M. Wise for useful discussions, and A. Cooray and M. Kesden for providing the results of their weak-lensing kurtosis calculations for comparison. K.S. acknowledges the support of the Canadian NSERC. This work was supported in part by NASA NAG5-9821, DOE DE-FG03-92-ER40701 and DE-FG03-02ER41215, and NSF PHY00-71856.
[1] P. Jordan, Naturwissenschaften 25, 513 (1937); Z. Phys. 113, 660 (1939).

[2] E. Teller, Phys. Rev. 73, 801 (1948).

[3] R.H. Dicke, Science 129, 621 (1959).

[4] K.P. Stanyukovich, Dokl. Akad. Nauk SSSR 147, 1348 (1962) [Sov. Phys. Dokl. 7, 1150 (1963)].

[5] R.H. Dicke, The Theoretical Significance of Experimental Relativity (Gordon and Breach, New York, 1965).

[6] G. Gamow, Phys. Rev. Lett. 19, 759 (1967); 19, 913 (1967).

[7] P. Forgács and Z. Horváth, Gen. Relativ. Gravit. 10, 931
(1979); 11, 205 (1979).

[8] J.D. Bekenstein, Phys. Rev. D 25, 1527 (1982).

[9] J.K. Webb et al., Phys. Rev. Lett. 87, 091301 (2001); J.K. Webb et al., Astrophys. Space Sci. 283, 565 (2003).

[10] J.N. Bahcall, C.L. Steinhardt, and D. Schlegel, astro-ph/0301507.

[11] J.-P. Uzan, Rev. Mod. Phys. 75, 403 (2003).

[12] R.A. Battye, R. Crittendon, and J. Weller, Phys. Rev. D 63, 043505 (2001).

[13] C.J.A.P. Martins et al., astro-ph/0302295. 
[14] H.B. Sandvik, J.D. Barrow, and J. Magueijo, Phys. Rev. Lett. 88, 031302 (2002); J.D. Barrow, H.B. Sandvik, and J. Magueijo, Phys. Rev. D 65, 063504 (2002); J. Magueijo, J.D. Barrow, and H.B. Sandvik, Phys. Lett. B 549, 284 (2002).

[15] D. Youm, Phys. Lett. 17A, 175 (2002).

[16] J.D. Barrow and D.F. Mota, Class. Quantum Grav. 20, 1 (2003).

[17] V.A. Kostelecky, R. Lehnert, and M.J. Perry, astro-ph/0212003.

[18] Q.-G. Huang and M. Li, J. High Energy Phys. 5, 026 (2003).

[19] G. Dvali and M. Zaldarriaga, Phys. Rev. Lett. 88, 091303 (2002).

[20] J.D. Bekenstein, Phys. Rev. D 66, 123514 (2002).

[21] T. Chiba and K. Kohri, Prog. Theor. Phys. 107, 631 (2002).

[22] K.M. Nollett and R.E. Lopez, Phys. Rev. D 66, 063507 (2002).

[23] G. Jungman et al., Phys. Rev. Lett. 76, 1007 (1996); Phys. Rev. D 54, 1332 (1996).

[24] P. de Bernardis et al., Nature (London) 404, 955 (2000); A.D. Miller et al., Astrophys. J. Lett. 524, L1 (1999); S. Hanany et al., ibid. 545, L5 (2000); N.W. Halverson et al., Astrophys. J. 568, 38 (2002); B.S. Mason et al., ibid. 591, 540 (2003); A. Benoit et al., Astron. Astrophys. 399, L2 (2003); J.H. Goldstein et al., astro-ph/0212517.

[25] D.N. Spergel et al., Astrophys. J., Suppl. Ser. 148, 175 (2003).

[26] M. Kamionkowski and A. Kosowsky, Annu. Rev. Nucl. Part. Sci. 49, 77 (1999).

[27] S. Hannestad, Phys. Rev. D 60, 023515 (1999).

[28] M. Kaplinghat, R.J. Scherrer, and M.S. Turner, Phys. Rev. D 60, 023516 (1999).

[29] P.J.E. Peebles, Astrophys. J. 153, 1 (1968).

[30] P. Boschán and P. Biltzinger, Astron. Astrophys. 336, 1 (1998).

[31] W.J. Karzas and R. Latter, Astrophys. J., Suppl. 6, 167 (1961); G.B. Rybicki and A.P. Lightman, Radiative Processes in Astrophysics (John Wiley \& Sons, New York, 1979).

[32] C.-P. Ma and E. Bertschinger, Astrophys. J. 455, 7 (1995).

[33] S.P. Goldman, Phys. Rev. A 40, 1185 (1989).

[34] G. Breit and E. Teller, Astrophys. J. 91, 215 (1940); J. Shapiro and G. Breit, Phys. Rev. 113, 179 (1959).
[35] S. Seager, D.D. Sasselov, and D. Scott, Astrophys. J., Suppl. Ser. 128, 407 (2000).

[36] P.J. Mohr and B.N. Taylor, Rev. Mod. Phys. 72, 351 (2000).

[37] D.J. Fixsen et al., Astrophys. J. 473, 576 (1996).

[38] G.F. Smoot et al., Astrophys. J. Lett. 396, L1 (1992).

[39] J. Kovac et al., Nature (London) 420, 772 (2002); A. Kogut et al., Astrophys. J., Suppl. Ser. 148, 161 (2003).

[40] W. Hu, N. Sugiyama, and J. Silk, Nature (London) 386, 37 (1997).

[41] J.R. Bond and G. Efstathiou, Mon. Not. R. Astron. Soc. 226, 655 (1987); A. Kosowsky, Ann. Phys. (N.Y.) 246, 49 (1996).

[42] M. Zaldarriaga and U. Seljak, Phys. Rev. D 55, 1830 (1997); M. Kamionkowski, A. Kosowsky, and A. Stebbins, ibid. 55, 7368 (1997); W. Hu and M. White, ibid. 56, 596 (1997).

[43] E. Newman and R. Penrose, J. Math. Phys. 7, 863 (1966); J.N. Goldberg et al., ibid. 8, 2155 (1967); K.S. Thorne, Rev. Mod. Phys. 52, 299 (1980).

[44] W. Hu, Phys. Rev. D 62, 043007 (2000).

[45] W. Hu, U. Seljak, M. White, and M. Zaldarriaga, Phys. Rev. D 57, 3290 (1998).

[46] U. Seljak and M. Zaldarriaga, Astrophys. J. 469, 437 (1996).

[47] U. Seljak, Astrophys. J. 463, 1 (1996); M. Zaldarriaga and U. Seljak, Phys. Rev. D 58, 023003 (1998).

[48] M. Kesden, A. Cooray, and M. Kamionkowski, Phys. Rev. D 66, 083007 (2002).

[49] Particle Data Group, K. Hagiwara et al., Phys. Rev. D 66, 010001 (2002).

[50] See, e.g., G.G. Raffelt, Phys. Rep. 198, 1 (1990); M.S. Turner, ibid. 197, 67 (1990).

[51] Y. Sortais et al., Phys. Scr. 95, 50 (2001).

[52] D.F. Mota and J.D. Barrow, astro-ph/0306047.

[53] A. Kurylov, K. Sigurdson, and M. Kamionkowski (in preparation).

[54] M. Kamionkowski and J. March-Russell, Phys. Lett. B 282, 137 (1992); Phys. Rev. Lett. 69, 1485 (1992); R. Holman et al., Phys. Lett. B 282, 137 (1992); R. Holman et al., Phys. Rev. Lett. 69, 1489 (1992).

[55] T. Banks, M. Dine, and M.R. Douglas, Phys. Rev. Lett. 88, 131301 (2002).

[56] N. Arkani-Hamed et al., Phys. Rev. Lett. 90, 221302 (2003). 